\title{
Comparison study of URANS and hybrid RANS-LES models on predicting vertical axis wind turbine performance at low, medium and high tip speed ratio ranges
}

T.P. Syawitri ${ }^{\mathrm{a}, \mathrm{b}}$, Y.F. Yao ${ }^{\mathrm{a}}$, B. Chandra ${ }^{\mathrm{a}}$ and J. Yao ${ }^{\mathrm{c}}$

${ }^{a}$ Department of Engineering Design and Mathematics, University of The West of England, Bristol BS16 1QY, United Kingdom

${ }^{b}$ Department of Mechanical Engineering, Universitas Muhammadiyah Surakarta, Surakarta 57162, Central Java, Indonesia

${ }^{c}$ School of Engineering, University of Lincoln, Brayford Pool, Lincoln LN6 7TS, United Kingdom

Corresponding author: yufeng.yao@uwe.ac.uk

\section{Highlights}

- Comprehensive analysis of URANS and hybrid RANS-LES CFD models for VAWT flow

- Study focuses on turbine power, moment coefficient and aerodynamics prediction

- Transition SST model is sufficient for overall performance prediction

- Hybrid RANS-LES can perform better prediction of VAWT dynamic stall behaviour

- $\quad$ SBES transitional SST generates more accurate predictions than other models

\section{ABSTRACT}

Comparison study of unsteady Reynolds-averaged Navier-Stokes (URANS) and hybrid RANS-LES (large-eddy simulation) models is carried out for predicting the performance of three-straight-bladed vertical axis wind turbine (VAWT) operating within three tip speed ratios $(T S R s)$ ranges, i.e. low $(1.44-2)$, medium $(2-2.64)$ and high $(2.64-3.3)$, respectively. The evaluation is focused on power coefficient, moment coefficient and vortex structure generation, growth and transportation predictions. It was found that URANS turbulence modelling is sufficient for averaged power coefficient $\left(C_{\text {pave }}\right)$ prediction and specific range of TSRs evaluation, and it also benefits from short simulation run time. To further evaluate flow field details at all TSR ranges and to understand the underlying flow physics such as dynamic stall behaviour, hybrid RANS-LES turbulence modelling is necessary. Comparing between hybrid 
models adopted, stress-blended eddy simulation (SBES) based on transition shear-stress transport (TSST) model has achieved an overall better performance, such as a reduction of simulation discrepancy by $50 \%$ in low TSR range compared to TSST turbulence model. In both medium and high TSR ranges, the modelling discrepancies are less than 3\% compared to TSST turbulence models at about $25 \%$ extra computational time. Furthermore, in high TSR range, hybrid RANS-LES models are able to predict the appearance of vortex shedding at high azimuthal angles $\left(\theta \geq 180^{\circ}\right)$ for which URANS models failed to capture.

Keywords: vertical axis wind turbine; computational fluid dynamics; turbulence model assessment; hybrid RANS-LES; stress-blended eddy simulation.

\section{Introduction}

With the advancement of computer power and the development of new algorithms, there is a growing trend to perform numerical study as a precursor step to expensive experiment for vertical axis wind turbine (VAWT) performance evaluation, and various approaches have been adopted, such as vortex model, blade element momentum (BEM) based models (i.e. multiple stream-tube model), and more recently computational fluid dynamics (CFD). The vortex model [1] and multiple stream-tube model [2] are two popular analytical models that have been used in VAWT model simulations. These two models are based on one-dimensional simplified governing equations that require some measurement data such as lift and drag coefficients of the employed aerofoils. However, these analytical models have exhibited poor accuracy when the aerofoil experiences dynamic stall due to inaccurate formulations to predict the tip vortex and dynamic stall effects [3]. Despite of higher costs and longer computational time, CFD technique of solving Navier-Stokes equations has become a powerful tool to design, analyse, and optimise VAWT performances. Overall, CFD can produce more accurate prediction of flow characteristics around wind turbines compared to the other numerical models [4].

Subject to its tip speed ratio (TSR), VAWT will experience different flow behaviours and unsteadiness during the operation. Therefore, choosing a proper computational domain, turbulence model and defining other numerical settings that can produce relatively small discrepancy for all TSR ranges operation is time consuming and sometime challenging. In general, flow behaviour around VAWTs can be analysed over three TSR ranges, namely low $T S R \mathrm{~s}$, medium TSRs (where an optimum TSR is usually obtained) and high TSRs, respectively. Comparing to medium and high TSRs ranges, it is more difficult to predict the performances 
and flow behaviour of VAWTs accurately in low TSRs range, mainly because the VAWT blades will experience local angles of attack $(A o A)$ higher than the stall $A o A$ for most azimuthal angles. Therefore, the blade can only generate either a very small or even a negative torque [5].

It is known that the flow surrounded VAWTs contains high level of unsteadiness, some are inherited from incoming background flows. Malael et al. categorized the unsteadiness level in low TSRs range as the second level of unsteadiness which is related to unsteady phenomenon called dynamic stall with drag reduction [6]. Practically, VAWT blades in this TSRs range will experience two types of dynamic stall. These two stalls are related to the existence of two peaks in the lift coefficient curve of non-rotating aerofoil operated in low TSRs range. The first stall is so-called lift dynamic stall that is dependent on the Reynolds number $(R e)$ and also due to a combination effect of the boundary layer separation and the unsteady (rotating) motion of the aerofoil [7]. The second stall is so-called drag dynamic stall which is independent on the Reynolds number. This stall only exists when the blades are operating in a narrow flow field. The VAWT blades will act as a force machine to move separated volume of air towards the blade radial direction. This term is also used to describe the delay in the drop of the second static lift coefficient, whilst a rotating blade passing through the downwind period of an upstream blade (i.e. azimuthal angle $\geq 180^{\circ}$ ) [6]. Drag dynamic stall will generate a small lift component but with a significant drag reduction over a short period of time in low TSRs range. This is a key factor in the continuity of torque production which is directly related to selfstarting capability of VAWTs. Therefore, it is very important to perform CFD modelling which can accurately predict VAWTs performance, especially in low TSRs range.

Despite of its ability to produce more accurate simulation results, large-eddy simulation (LES) model is rarely used in CFD simulation of VAWTs due to its expensive computational cost and extremely long run time. Majority of numerical studies nowadays are still using unsteady Reynolds-averaged Navier-Stokes (i.e. unsteady RANS or URANS in short) models which are very sensitive to numerical settings and model choice. After comparing several URANS turbulence models, Rezaeiha et al. concluded that transition shear-stress transport (TSST) turbulence model is preferable for VAWTs simulation as it can predict the laminar-toturbulent transition, which likely occurs on the VAWT blade surfaces [8]. However, while the predicted averaged power coefficients over one turbine revolution $\left(C_{\text {pave }}\right)$ were close to the experimental data in the low TSRs range, it still over-predicted the $C_{\text {pave }}$ value in the high TSRs range. The cause of this discrepancy has not been fully understood yet [8].

To compromise numerical accuracy and computational cost, hybrid RANS-LES models are suggested and employed by some researchers in VAWT applications ( [9], [10], [11] ). 
These models use URANS approach to model the small-scale eddies near the wall boundary, while the large-scale eddies in the far field and around flow separation regions are simulated using LES model [11]. As such, this technique is able to produce more accurate results than URANS models and in the meantime, to overcome the obstacle of requiring enormous computational resources of applying LES model for whole computational domain.

Even though there are some reports on the use of hybrid RANS-LES turbulence models for CFD simulation of VAWT flows, based on authors' knowledge, there is no comprehensive investigation to assess the capability of hybrid RANS-LES turbulence models for predicting VAWTs performance compared to URANS turbulence models for low-to-high ranges TSRs operation. Mostly, they were only comparing one hybrid RANS-LES turbulence model with a few URANS turbulence models or even only one URANS turbulence model. They did not provide direct comparison between several hybrid RANS-LES turbulence models. Moreover, they usually compared the $C_{\text {pave }}$ predictions only rather than the blade instantaneous moment coefficients and the details of blade aerodynamics. In addition, they did not present the effects of turbulence models on the accuracy of predicting VAWTs performance for all ranges of TSRs operation and the reason(s) behind them.

Therefore, in this study, a comparison study is performed for the accuracy of VAWTs simulation of three different hybrid RANS-LES models, namely delayed-detached eddy simulation (DDES), improved-delayed-detached eddy simulation (IDDES) and a relatively new hybrid RANS-LES model stress-blended eddy simulation (SBES), along with three URANS turbulence models, namely $k-\varepsilon$ realizable with enhanced wall treatment, $k-\omega$ SST and Transition SST models, respectively. Note that, in the authors' knowledge, this is the first attempt to apply SBES in CFD simulation of VAWT. All CFD simulations are carried out using ANSYS Fluent v19 [12]. The comparison of revolution convergences between hybrid RANSLES and URANS turbulence models is undertaken after some precursor studies such as grid convergence and time independence, due to the importance of obtaining statistically converged flow field before collecting data samples in VAWTs simulation. Then, the $C_{\text {pave }}$ results of CFD simulation are compared with the results of wind tunnel experiment by Castelli et al. [13] and are analysed for considered TSRs operation range in this study, i.e. low TSRs range of $1.44-$ 2, medium TSRs range of $2-2.64$ (with an optimum TSR of 2.64) and high TSRs range of 2.64 - 3.3, respectively. Distribution of instantaneous moment coefficients and turbine aerodynamics flow field are only compared with the results of CFD simulations, as Castelli et al. [13] did not provide the experimental results in these regards. The detailed comparison of this study will help to understand the performance of hybrid RANS-LES in VAWTs simulation 
compared to URANS turbulence model, in particular the new SBES hybrid RANS-LES turbulence model [14], in all three ranges of TSRs operation.

\section{Vertical Axis Wind Turbine (VAWT) Characteristics}

The study is based on experimental and numerical studies by Castelli et al. [13] which have been widely used as validation case in many numerical studies of VAWTs $[8,15,16,17]$. The experiment considered a three-straight-bladed Darrieus VAWT equipped with NACA 0021 aerofoils in TSRs ranging between 1.44 and 3.3 as shown in Figure 1. The trailing edge of each blade is defined as straight wall with a finite thickness of $0.38 \mathrm{~mm}$. The turbine aspect ratio is 1.4. The free stream velocity $\left(U_{\infty}\right)$ is set to be $9 \mathrm{~m} / \mathrm{s}$. The turbine rotational speed $\left(\omega_{t}\right.$ $(\mathrm{rad} / \mathrm{s}))$ is calculated based on Equation (1) below

$$
T S R=\frac{\omega_{t} R}{U_{\infty}}
$$

where $R(m)$ is the turbine radius.

The main geometrical features and the operational parameters of the test VAWT (both numerical and experimental models) can be found in Table 1. Note that, Castelli et al. [12] have also compared their three-dimensional (3D) experiment with two-dimensional (2D) CFD simulation in regard of power coefficient. They argued that the difference between 3D experiment and 2D CFD was mainly caused by the combined effects of finite blade length and spoke drag.

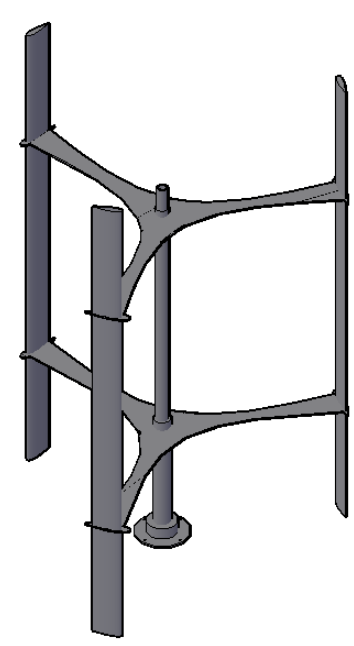

(a) 

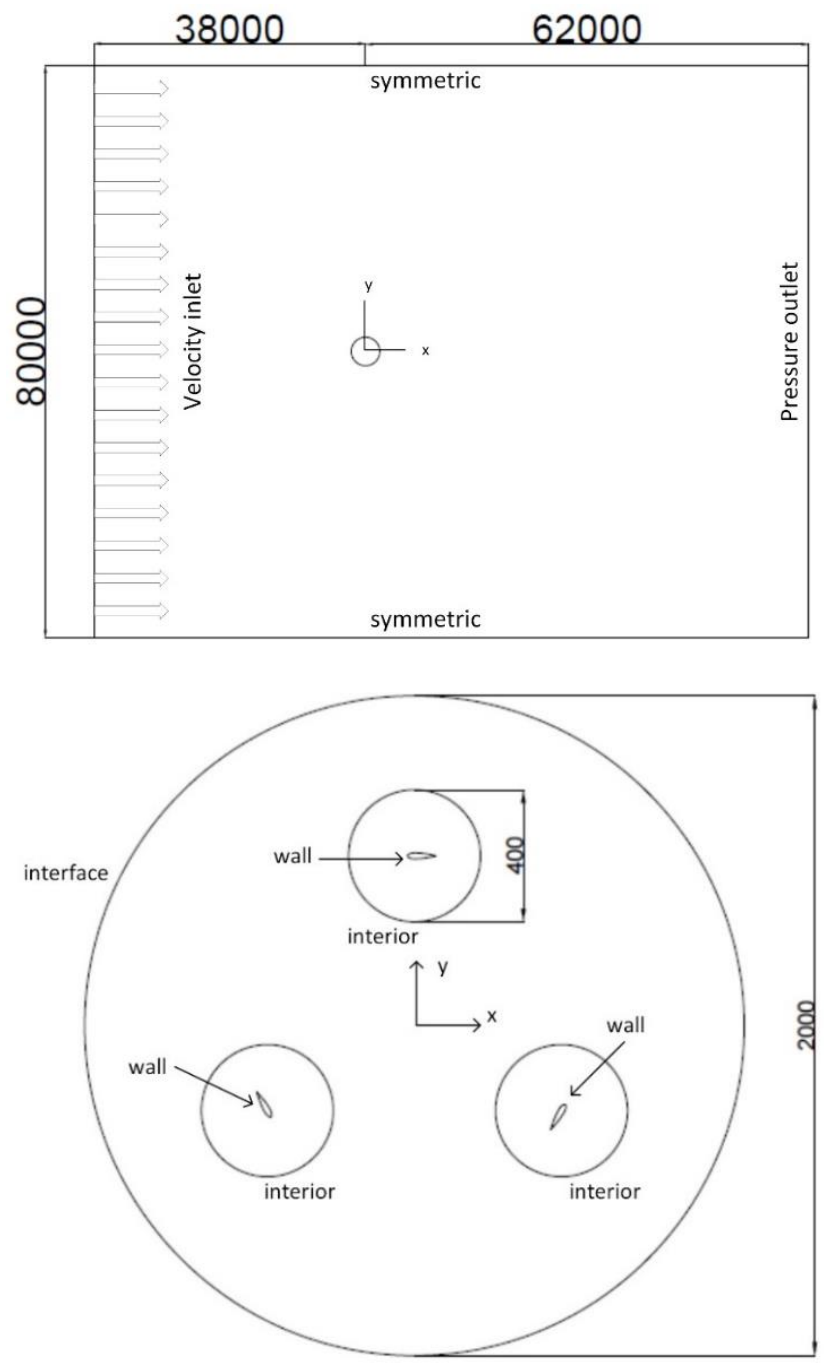

(b)

Figure 1. VAWT model based on Castelli et al. [13], (a) 3D experimental and (b) 2D computational domain (all measurements are in $\mathrm{mm}$ ).

Table 1. Main geometrical features of Castelli et al. model [13].

\begin{tabular}{|c|c|c|}
\hline Parameters & Simulation & Experiment \\
\hline VAWT diameter $\left(D_{\text {rotor }}(\mathrm{mm})\right)$ & 1030 & 1030 \\
\hline Blade height $\left(H_{\text {rotor }}(\mathrm{mm})\right)$ & 1000 (for 2D simulation) & 1456.4 \\
\hline VAWT swept area $\left(A_{s}\left(\mathrm{~m}^{2}\right)\right)$ & 1.03 & 1.236 \\
\hline Number of blade $(N(-))$ & 3 & 3 \\
\hline Blade profile & NACA 0021 & NACA 0021 \\
\hline Chord length $(c(m m))$ & 85.8 & $0.5 \mathrm{c}$ \\
\hline Spoke-blade connection & $0.25 \mathrm{c}$ & 0.5 \\
\hline solidity $(\sigma(-))$ & 0.5 & \\
\hline
\end{tabular}




\section{CFD Simulation}

\subsection{Simulation domain and grid decomposition}

Figure 2 illustrates a 2D computational domain which consists of a rotating core where three turbine blades are located inside it and a rectangular far field domain surrounding the rotating core. The current CFD mesh uses hybrid mesh type and has a total of 117,353 elements and 174 elements around each blade surface with refined meshes towards both the leading and trailing edge regions, respectively.

\subsubsection{Rectangular far field domain}

A rectangular domain is used for the far field. As suggested by Wang et al. [16], both inlet and outlet planes are placed at 40 rotor diameters away from the centre, while the side walls are placed at 20 rotor diameters away from the centre of turbine rotational axis so numerical boundary conditions will have negligible influences on simulation results. The inlet and outlet are set as velocity inlet and pressure outlet conditions, respectively. Meanwhile, symmetric boundary conditions are used for the side walls. A structured grid with quadrilateral cells is generated for this non-rotating domain (see Figure 3a). This domain has overall 34,200 elements.

\subsubsection{Rotating core}

The circular rotating core is treated as the fluid region representing the revolution of VAWTs. As mentioned in the previous studies $[18,19]$, the size of rotating core needs to be at least around 1.5 - 2 times of turbine diameter to avoid unwanted disturbances produced by the sliding interface. In this study, the size of rotating core is kept the same as those well-validated studies, which is $2000 \mathrm{~mm}$ (about 1.94 times of turbine diameter).

The domain, together with meshes inside, rotates in an anticlockwise direction around the turbine rotational axis at a given angular velocity. The mesh moves at the prescribed speed for this simulation. Therefore, the mesh motion is only applied in this sub-domain. In general, between the far field domain and the rotating core sub-domain, mesh topologies and even sizes could be different, and thus to ensure the continuity of fluid flow across the interface, a sliding boundary condition is set at the interface between these two domains, together with data interpolations of same or higher order than numerical scheme. The rotating core sub-domain uses quadrilateral dominant elements (see Figure 3b) with a total number of 20,513 elements. 


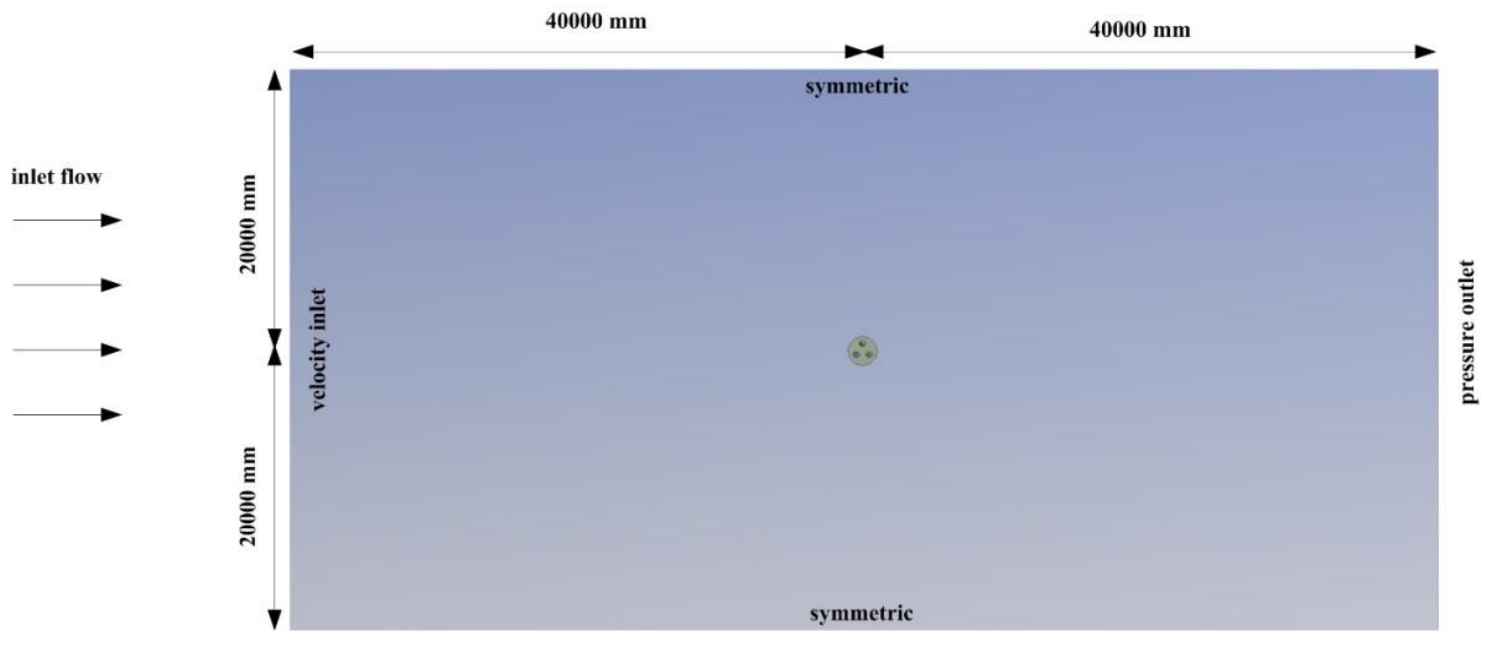

(a) Overall computational domain

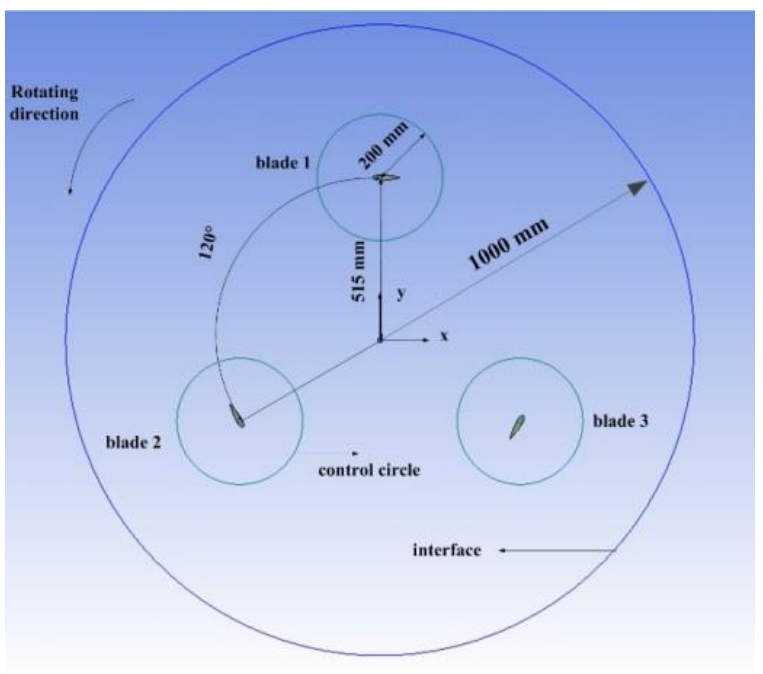

(b) Rotating core sub-domain

Figure 2. Detailed 2D computational domain and sub-domains.

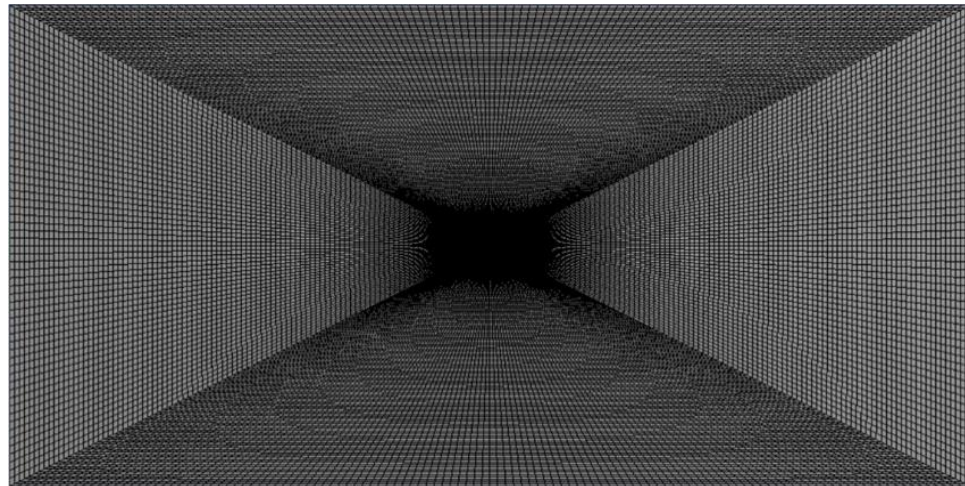

(a) Far field

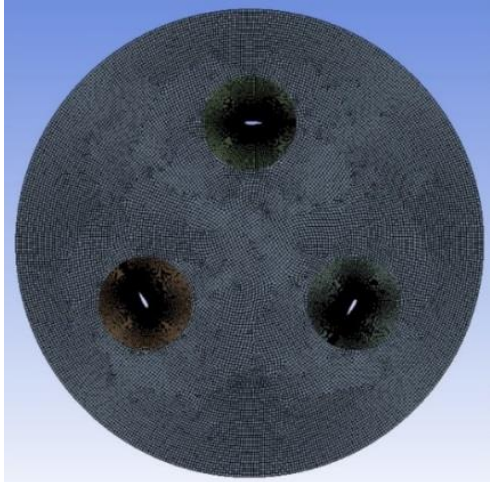

(b) Rotating core

Figure 3. Grid details in two main sub-domains. 


\subsubsection{Control circle}

Three control circles with embedded blades are placed inside the rotating core separated by $120^{\circ}$ angular distance to each other. Each control circle has a radius of $200 \mathrm{~mm}$, in which a structured O-type grid discretization around the blade is produced. Total elements in each control circle are 20880. The control circle boundary is set as 'interior' to ensure the continuity of the fluid flow. Blade surfaces are also defined as rotating walls with reference to the rotation centre, i.e. the main rod of the spoke-blade-connection on each blade, to ensure it rotates together with the rotating core sub-domain.

The grid discretization in this sub-domain uses structured quadrilateral elements with fine grids in the near wall region (see Figure 4). When applying turbulence model to solve nearwall region, the first grid must be placed inside the viscous sublayer. Hence, the normal distance of the first element to the wall must be pre-defined so that it satisfies the criteria of the non-dimensional wall distance of $y^{+}<1$ (for SST turbulence model). As the wall function is not applicable for flows which experience flow separation like flow around the blade of VAWT, it is important to maintain $y^{+}$value lower than unity to properly resolve the laminar sublayer region near the wall [20]. Note that, when generating grids for DDES and IDDES turbulence models, LES criteria in far field region is applied. Hence, this study has two grid types. The first grid type is the one used for DDES and IDDES turbulence models which has higher number of elements to accommodate the LES criteria in far field region and the second grid type is the one used for URANS turbulence models in the rest of flow domain.

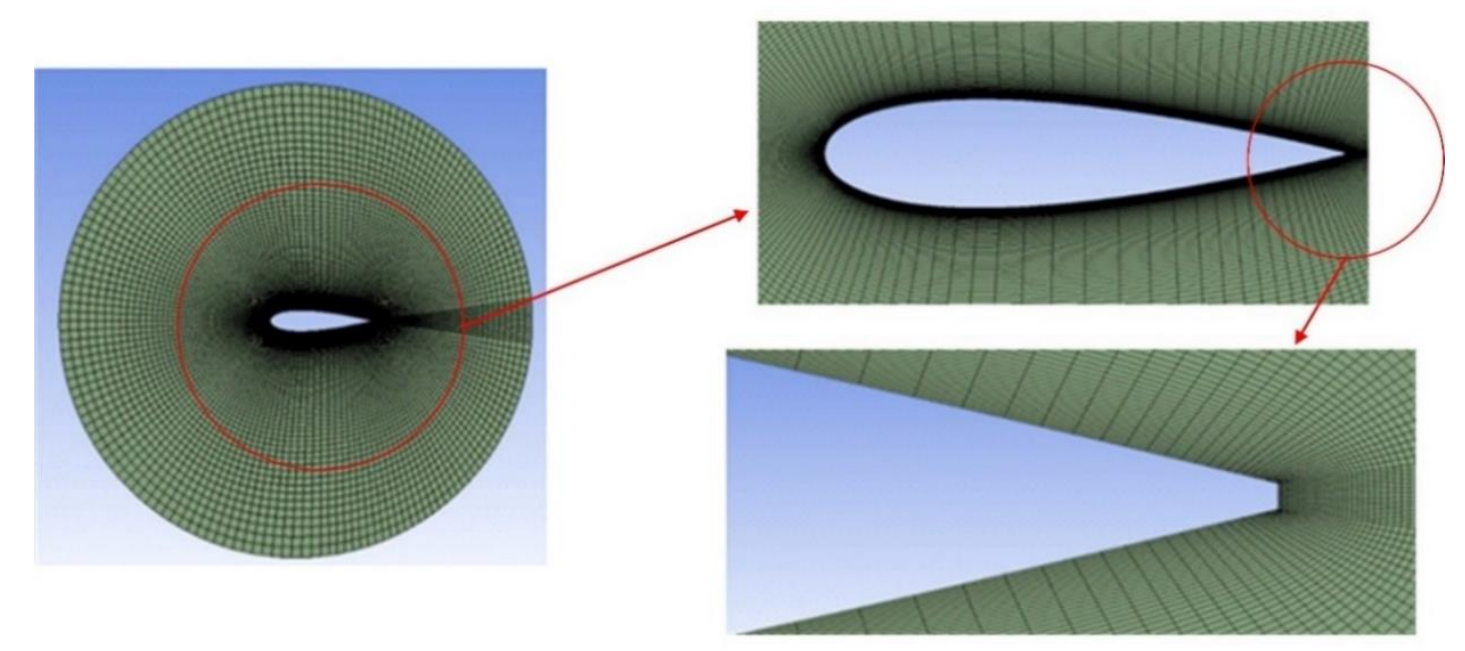

Figure 4. Detailed grid around the blade wall. 


\subsection{Turbulence models}

\subsubsection{URANS turbulence models}

This study considers two two-equation models and one four-equation models with transition model to accommodate the laminar-to-turbulent transition process.

1) Two-equation $k-\varepsilon$ realizable with enhanced wall treatment (RKE)

This model has proven to have better performance for flow prediction compared to standard $k-\varepsilon$, especially for flow with primary separation and secondary flow features, due to its better ability to capture the mean flow. This is mainly due to the use of non-constants in turbulence viscosity $\left(\mu_{t}\right)$ formulation. Equations (2) and (3) show the formulation of turbulence viscosity in this model. It can be seen that the $C_{\mu}$ is no longer a constant but a variable and the turbulence energy dissipation rate, $\varepsilon\left(\mathrm{m}^{2} / \mathrm{s}^{3}\right)$, is derived from an exact equation for the transport of the mean-square vorticity fluctuation [21].

$$
\begin{aligned}
\mu_{t} & =\rho C_{\mu} \frac{k^{2}}{\varepsilon}, \\
C_{\mu} & =\frac{1}{A_{0}+A_{s} \frac{k U^{*}}{\varepsilon}},
\end{aligned}
$$

where $\rho$ is fluid density $\left(\mathrm{kg} / \mathrm{m}^{3}\right)$ and $k$ is turbulence kinetic energy $\left(\mathrm{m}^{2} / \mathrm{s}^{2}\right)$. In equation (3), $U^{*} \equiv \sqrt{S_{i j} S_{i j}+\widetilde{\Omega_{l j}} \widetilde{\Omega_{l j}}}$ is the modified streamwise mean with $S_{i j}=\frac{1}{2}\left[\frac{\partial U_{i}}{\partial x_{j}}+\frac{\partial U_{j}}{\partial x_{i}}\right]$ is strain rate and $\widetilde{\Omega_{\imath \jmath}}$ is the modified vorticity tensor as explained by Shih et al [22].

This model is preferred than other $k-\varepsilon$ models on single moving reference frame systems associated with computational domain that contains both rotating and stationary fluid zones. However, as RKE model sometimes generates non-physical turbulence viscosities in this kind of computational domain, its application on multiple reference frame systems like VAWT model needs to be considered carefully. In addition, this model also inherits the poor performance of any $k-\varepsilon$ based model for flows with low Reynolds number (Re) [23]. In this study, the enhanced wall treatment for near-wall modelling is also included to make sure that the flow in viscous sublayer can be captured.

2) Two-equation Shear-Stress Transport $k$ - $\omega$ (SST)

This model is combining some key features of $k-\varepsilon$ and standard $k-\omega$ models. It applies $k$ $\omega$ formulation [24] in the inner parts of the boundary layer and switches to $k-\varepsilon$ formulation [25] in the region away from the wall. Hence, this turbulence model has capability to predict flow at low $R e$ without the need of using any extra dumping functions and thus can avoid the 
common problem of high sensitivity to the inlet free stream turbulence in a standard $k-\omega$ model. The turbulence viscosity equation is modified by adding a limiter to avoid over prediction of the eddy-viscosity (see Equations (4) and (5)).

$$
\begin{aligned}
& \mu_{t}=\frac{\rho k}{\omega} \frac{1}{\max \left[\frac{1}{\alpha^{*}+\frac{S F_{2}}{a_{1} \omega}}\right]} \\
& \alpha^{*}=\alpha_{\infty}^{*}\left\lceil\frac{\alpha_{0}^{*}{ }^{R e_{t}} / R_{k}}{1+{ }^{R e} t / R_{k}}\right\rceil,
\end{aligned}
$$

where $\omega$ is specific dissipation rate $(1 / s), \alpha^{*}$ is low $\operatorname{Re}$ correction parameter, $S=\sqrt{2 S_{i j} S_{i j}}$ is shear strain rate, $\alpha_{1}=0.31, \alpha_{\infty}^{*}=1, \alpha_{0}^{*}=\frac{\beta_{i}}{3}, \beta_{i}=0.072, R e_{t}=\frac{\rho k}{\mu \omega}$ is viscosity ratio, $R_{k}=6$. Note that, for high-Re number flow, $\alpha^{*}=\alpha_{\infty}^{*}=1$. Furthermore, $F_{2}=\tanh \left(\Phi_{2}^{2}\right)$ is the blending function and $\Phi_{2}$ is the set of constants in the transformed $k-\varepsilon$ model which defined as $\Phi_{2}=\max \left\lceil 2 \frac{\sqrt{k}}{0.09 \omega y}, \frac{500 \mu}{\rho y^{2} \omega}\right\rceil$ with $y$ is the distance to the next wall surface $(m)$ and $\mu$ is dynamic viscosity $\left(\mathrm{m}^{2} / \mathrm{s}\right)$.

Moreover, the existence of transport of the turbulence shear stress in turbulence viscosity equation (see Equation (5)) makes this model having better performance for flow with adverse pressure gradient and separation such as aerofoil flow at high AoA. As the Reynolds number in this study is relatively low, low Re corrections model can be activated.

In addition, Menter [24] and Kato-Launder [26] production limiters are also activated to prevent enormous turbulence energy production in the stagnation region. The Menter's production limiter introduces a parameter $C_{\text {lim }}$ (i.e. coefficient of limit) in the equation of turbulence energy generation $\left(G_{k}\right)$ of the model (see Equations (6) and (7)). Thus, the model will choose the minimal value between the calculated turbulence energy and a prescribed limit value to avoid over excessive turbulence kinetic energy production.

$$
\begin{gathered}
G_{k}=\min \left[G_{k, c a l}, C_{l i m} \rho \varepsilon\right], \\
G_{k, c a l}=\mu_{t} S^{2},
\end{gathered}
$$

where coefficient $C_{\text {lim }}$ has a default value of 10 . There is no effect of this limiter on the shear layer performance of the model, but it avoids the 'artificial' stagnation point in aerodynamic simulations. 
Alternatively, Kato-Launder modified the equation of turbulence energy generation by replacing shear strain rate with the vorticity rate as shown in Equation (8). This modification is based on the observation that the excessive level of turbulence kinetic energy production is caused by a very high level of shear strain rate in the stagnation regions [26]. This enormous level of shear strain rate is mainly induced by nearly irrotational flow with a very small vorticity rate in the flow field near the stagnation point.

$$
G_{k, c a l}=\mu_{t} S \Omega,
$$

where $\Omega=\sqrt{\Omega_{i j} \Omega_{i j}}$ is the magnitude of the vorticity tensor.

3) Four-equation transition SST (TSST)

This model is based on the SST $k-\omega$ transport equations with addition of two other transport equations: intermittency $(\gamma)$ (see Equation (9)) and transition onset criteria in term of momentum-thickness Reynolds number $\left(\operatorname{Re}_{\theta}\right)$ (see Equations (10) to (13)). The intermittency can trigger local transition while the transition onset $\operatorname{Re}_{\theta}$ will enable the model to capture the non-local influence of the change of turbulence intensity due to decay of the turbulence kinetic energy in the free stream and free stream velocity variation outside of the wall boundary layer [27]. Due to the present of these two additional equations, prediction of the transition onset and its length can be dealt with by SST $k-\omega$ model. Based on previous works [27, 28, 29], this model was proven to have good accuracy on predicting natural and/or induced separation and bypass transitions for wall-bounded flows. In this study, the turbulence production limiters proposed by Menter [24] and Kato and Launder [26] are activated to avoid the excessive turbulence energy generation and very high level of shear strain rate in the stagnation region.

$$
\frac{\partial(\rho \gamma)}{\partial y}+\frac{\partial\left(\rho U_{j} \gamma\right)}{\partial x_{j}}=P_{\gamma 1}-E_{\gamma 1}+P_{\gamma 2}-E_{\gamma 2}+\frac{\partial}{\partial x_{j}}\left[\left(\mu+\frac{\mu_{t}}{\sigma_{\gamma}}\right) \frac{\partial \gamma}{\partial x_{j}}\right]
$$

In Equation (15), $P_{\gamma 1}=C_{a 1} F_{\text {length }} \rho S\left[\gamma F_{\text {onset }}\right]^{C_{\gamma 3}}$ and $E_{\gamma 1}=C_{e 1} P_{\gamma 1} \gamma$ are the transition sources with $C_{a 1}=2, F_{\text {length }}$ is an empirical correlation that controls the length of the transition region, $F_{\text {onset }}$ is functions that are used to trigger the intermittency production, $C_{\gamma_{3}}=0.5$, and $C_{e 1}=1 . P_{\gamma 2}=C_{a 2} \rho \Omega F_{\text {turb }}$ and $E_{\gamma 2}=C_{e 2} P_{\gamma 2} \gamma$ are destruction/relaminarisation with $F_{\text {turb }}=$ $e^{-\left(\frac{R_{t}}{4}\right)^{4}}$ is the function to control the length of turbulence region. 


$$
\begin{gathered}
\frac{\partial\left(\rho \widetilde{R e_{\theta t}}\right)}{\partial y}+\frac{\partial\left(\rho U_{j} \widetilde{R e_{\theta t}}\right)}{\partial x_{j}}=P_{\theta t}+\frac{\partial}{\partial x_{j}}\left[\sigma_{\theta t}\left(\mu+\mu_{t}\right) \frac{\partial \widetilde{R e_{\theta t}}}{\partial x_{j}}\right] \\
P_{\theta t}=C_{\theta t}^{\frac{\rho}{t}}\left(R e_{\theta t}-\widetilde{R e_{\theta t}}\right)\left(1.0-F_{\theta t}\right) \\
F_{\theta t}=\min \left(\max \left(F_{\text {wake }} e^{\left(-\frac{y}{\delta}\right)^{4}}, 1.0-\left(\frac{\gamma-1 / 50}{1.0-1 / 50}\right)^{2}\right), 1.0\right), \\
F_{\text {wake }}=e^{-\left(\frac{R e_{\omega}}{1 E+5}\right)^{2}},
\end{gathered}
$$

where $R e_{\theta t}$ is transition onset momentum thickness Reynolds number, $\widetilde{R e_{\theta t}}$ is local transition onset momentum thickness Reynolds number, $P_{\theta t}$ is the source term, $\sigma_{\theta t}=2.0, t=\frac{500 \mu}{\rho U^{2}}$ is a time scale that is present for dimensional reasons and $C_{\theta t}=0.03 . F_{\theta t}$ in Equation (11) is blending function that is used to turn off the source term in the boundary layer, $F_{\text {wake }}$ is a function to ensures that the blending function is not active in the wake regions downstream of the body, $\delta=\frac{50 \Omega y}{U} \delta_{B L}$ is boundary layer thickness $(m), \delta_{B L}=\frac{15}{2} \theta_{B L}$ is boundary layer transition thickness $(m), \theta_{B L}=\frac{\widetilde{R e_{\theta} t} \mu}{\rho U}$ is momentum thickness in boundary layer transition $(m)$, and $R e_{\omega}=\frac{\rho \omega y^{2}}{\mu}$ is dissipation Reynolds number.

\subsubsection{Hybrid RANS-LES turbulence models}

Three types of hybrid RANS-LES turbulence models are applied in this study, of which the first is based on delayed-detached eddy simulation (DDES) model, the second is based on improved-detached-delayed eddy simulations (IDDES), and the third is a relatively new model called stress-blended eddy simulation (SBES).

\section{1) Delayed-Detached Eddy Simulation (DDES)}

This model has based on DES model which treats the attached boundary layer using RANS while LES is only implemented in the separated flow regions. DDES is designed to solve some problems in standard DES model such as high grid-size sensitivity leading to grid induced separation (GIS) which is caused by "grey area" where the models vary from URANS to LES and possible delay in the formation of instabilities in mixing layers [30]. This model has been successful in overcoming some drawbacks in DES model, but at extra computational cost due to extreme grids used in the model [31]. This study chooses SST $k$ - $\omega$ model for URANS simulation, therefore this model applies DES equations proposed by Menter and Kuntz [30] which modified the dissipation term of the turbulence kinetic energy by 
$Y_{k}=\rho \beta^{*} k \omega F_{D E S}$,

where $\beta^{*}$ is a model constant $=0.09$ and the blending function, $F_{D E S}$, is expressed as

$F_{D E S}=\max \left(\frac{L_{t}}{C_{D E S} \Delta_{\max }}, 1\right)$

where $C_{D E S}$ is a calibration constant used in the DES model and has a value of $0.61, \Delta_{\max }$ is the grid spacing $(m)$ and $L_{t}$ is turbulence length scale $(m)$ which is calculated by

$L_{t}=\frac{\sqrt{k}}{\beta^{*} \omega}$,

To protect the boundary layer from GIS, a shielded function is added into the equation of blending function of DES, therefore it becomes

$F_{D E S}=\max \left(\frac{L_{t}}{C_{D E S} \Delta_{\max }}\left(1-f_{D D E S}\right), 1\right)$

where $f_{D D E S}$ is the blending function used in the DDES model and is expressed by

$f_{D D E S}=\tanh \left[\left(C_{d 1} r_{d}\right)^{C_{d 2}}\right]$

where $C_{d 1}=20$ and $C_{d 2}=3$ and

$r_{d}=\frac{v_{t}+v}{\kappa^{2} y^{2} \sqrt{0.5\left(S^{2}+\Omega^{2}\right)}}$

where $v_{t}$ is turbulence kinematic viscosity $\left(\mathrm{m}^{2} / \mathrm{s}\right), v$ is laminar kinematic viscosity $\left(\mathrm{m}^{2} / \mathrm{s}\right)$, and Karman constant $\kappa=0.41$.

This model has been proven to be able to produce good prediction in rotating flow with separation and flow around aerofoil with stall condition [32]. Nevertheless, as this is a DES type turbulence model, refined grids are still needed not only near the wall but also in the far field regions. In this study, DDES is deployed with SST $k-\omega$ for RANS model with the activation of low Reynolds number correction model. 


\section{2) Improved-Detached-Delayed Eddy Simulations (IDDES)}

It is known that DES and DDES will deal with two parts of the entire logarithmic layer (denote 'log-layer' thereafter) in a separate manner, i.e. “inner” log-layer part by RANS model and "outer" log-layer part by LES model. The LES function will only become activate in regions with local grid-sizes that are much smaller than the distance of this region to the wall. However, these two layers usually mismatched in DES and DDES models causing underprediction of skin friction coefficient by $15-20 \%$ compared to the experiment results [33]. Therefore, IDDES is proposed to address this problem by including a non-zonal hybrid RANSLES model called wall-modelled LES (WMLES). It has new definition of the sub-grid lengthscale in which an explicit wall-distance dependence is included. This addition allows DESbased model to produce different response depending on the grid resolution and inflow turbulence content. WMLES will be activated if the grid resolution is fine enough and inflow turbulence exists in the simulation, contrarily it will operate DDES model [33]. IDDES model has been demonstrated to have good ability in predicting massive flow separation and vortex shedding such as in flow around aerofoil at high angle of attack $(A o A)$.

3) Stress-Blended Eddy Simulation (SBES)

DES-based hybrid RANS-LES models including DDES and IDDES have shown several shortcomings for industrial application, in particular, when the mesh is refined in the boundary layer region, an issue of so-called GIS may appear, and it is due to LES grid limiter influence on RANS model causing improper balancing between RANS and LES turbulence content (even when using DDES). Moreover, there is tendency towards slow "transition" from the RANS to the LES zones in separating shear layers (SSL) [34] with no clear differentiator between RANS and LES regions. Hence, Menter has proposed an upgraded version of DES model called SBES. This model revises the shielding function of the shielded DES (SDES) SST model to protect the RANS boundary layers and automatically switch to an existing algebraic LES model in the LES zone [14]. While the blending function remains the same as that of the shielding function SDES $\left(f_{S D E S}\right)$, in the LES zone where $f_{S D E S}=0$, SBES introduces an explicit model to switch to an algebraic LES. This modification changes the turbulence stress tensor and eddy viscosity equations as

$$
\begin{gathered}
\tau_{i, j}^{S B E S}=f_{S D E S} \tau_{i, j}^{R A N S}+\left(1-f_{S D E S}\right) \tau_{i, j}^{L E S}, \\
v_{t}^{S B E S}=f_{S D E S} v_{t}^{R A N S}+\left(1-f_{S D E S}\right) v_{t}^{L E S},
\end{gathered}
$$


where $\tau_{i, j}^{S B E S}, \tau_{i, j}^{R A N S}$ and $\tau_{i, j}^{L E S}$ are turbulence stress tensors and $v_{t}^{S B E S}, v_{t}^{R A N S}$ and $v_{t}^{L E S}$ are turbulence kinematic viscosities.

As a result, the RANS and the LES zones can be clearly distinguished by visualizing the shielding function. Moreover, due to the lower turbulence stress level enforced by the LES model, SBES model can change rapidly from the RANS to the LES function in SSL, which then can produce better, realistic, and consistent solutions. Furthermore, this turbulence model allows a RANS-LES “switch" being predicted even on a coarser grid that other DES models cannot. This model has been applied in the CFD simulations of rotating devices [35, 36, 37]. It was found that this model could generate better prediction compared to RANS and other hybrid RANS-LES models. SBES can produce finer turbulence structures and ordered and abundant vortex structures compared to RANS models. Meanwhile, it offers faster development of turbulence and more clear and ordered turbulence structures compared to other hybrid RANSLES models.

\subsection{Computational settings}

In this study, the incompressible URANS equations are solved using a coupled numerical scheme for pressure-velocity coupling and a second-order scheme for temporal and spatial discretization. In hybrid RANS-LES simulation, computational settings remain the same as URANS simulation except for momentum spatial discretization where Bounded Central Differencing is applied as the requirement of hybrid RANS-LES model. All residual convergence criterions for the inner loop are set to be equal to or less than $10^{-6}$.

The complex nature of VAWT flow has limited the ability for all parameters reaching the same residual criteria simultaneously during the simulation. In order to overcome this issue, previous study suggested that applying 40 sub-iterations in each time step could reduce the turbulence kinetic energy residuals by an order of $10^{-4}$ [18]. Hence, 40 sub-iterations per time step are applied in the simulations. Note that, for the initialization procedure, this study uses hybrid initialization which is a collection of recipes and boundary interpolation methods [12]. A Laplace equation is solved during the initialization to produce a velocity field that conforms to complex domain geometries, and also a pressure field which smoothly connects high and low pressure values in the computational domain [12]. 


\section{Spatial and Temporal Resolution Studies}

\subsection{Spatial (grid) convergence study}

Simulations for case study of $T S R=2.5$ were performed on coarse, medium and fine three successive grids (see Table 2) for grid convergence study using RKE turbulence model. The influence of element sizes around the blade is considered with 87, 174 and 348 grid elements, respectively for each simulation.

Table 2. Details of the number of elements for each grid

\begin{tabular}{|c|c|c|c|c|}
\hline Grid & \multirow{2}{*}{ Type of grid } & $\begin{array}{c}\text { Total } \\
\text { Elements }\end{array}$ & $\begin{array}{c}\text { Elements around } \\
\text { blade wall }\end{array}$ & $\begin{array}{c}\text { Trailing edge } \\
\text { elements }\end{array}$ \\
\cline { 1 - 3 } Coarse & & 86033 & 87 & 7 \\
\cline { 1 - 1 } Medium & \multirow{2}{*}{ Structured grid } & 117353 & 174 & 14 \\
\cline { 1 - 1 } Fine & & 179993 & 348 & 28 \\
\hline
\end{tabular}

Figure 5 illustrates the comparison of moment coefficients $\left(C_{m}\right)$ over one revolution using different grids. Note that, the $C_{m}$ value here is extracted at revolution after the difference between the latest revolution and the previous revolution is less than $1 \%$. The calculation of $C_{m}$ is defined by Equation (22),

$$
C_{m}=\frac{M}{\frac{1}{2} \rho U_{\infty}^{2} A L},
$$

where the moment $M(N)$ is from the simulation, $U_{\infty}(\mathrm{m} / \mathrm{s})$ is the free stream velocity of the simulated model, $A\left(\mathrm{~m}^{2}\right)$ is the area of the simulated model and $L(m)$ is the distance of the simulated model to the rotating axis. The time history of this coefficient can be monitored and analysed. Based on obtained $C_{m}, C_{p}$ can be calculated by Equation (23),

$$
C_{p}=T S R \times C_{m}
$$

Similar to the baseline 2D VAWT CFD study, the area $(A)$ is defined as the rotor swept area $\left(A_{s}\right)$ and the length $(L)$ is set to be the same as the rotor radius $(0.515 \mathrm{~m})$ for 2D CFD.

The curves representing moment coefficient at varying azimuthal position have shown little difference between results from the medium and fine grids while coarse grid could not produce satisfying instantaneous moment coefficients. Moreover, the relative discrepancy of 
averaged power coefficient over one turbine revolution $\left(C_{\text {pave }}\right)$ between the medium and fine grids is less than $0.02 \%$. Therefore, the medium grid is chosen for the rest of simulations.

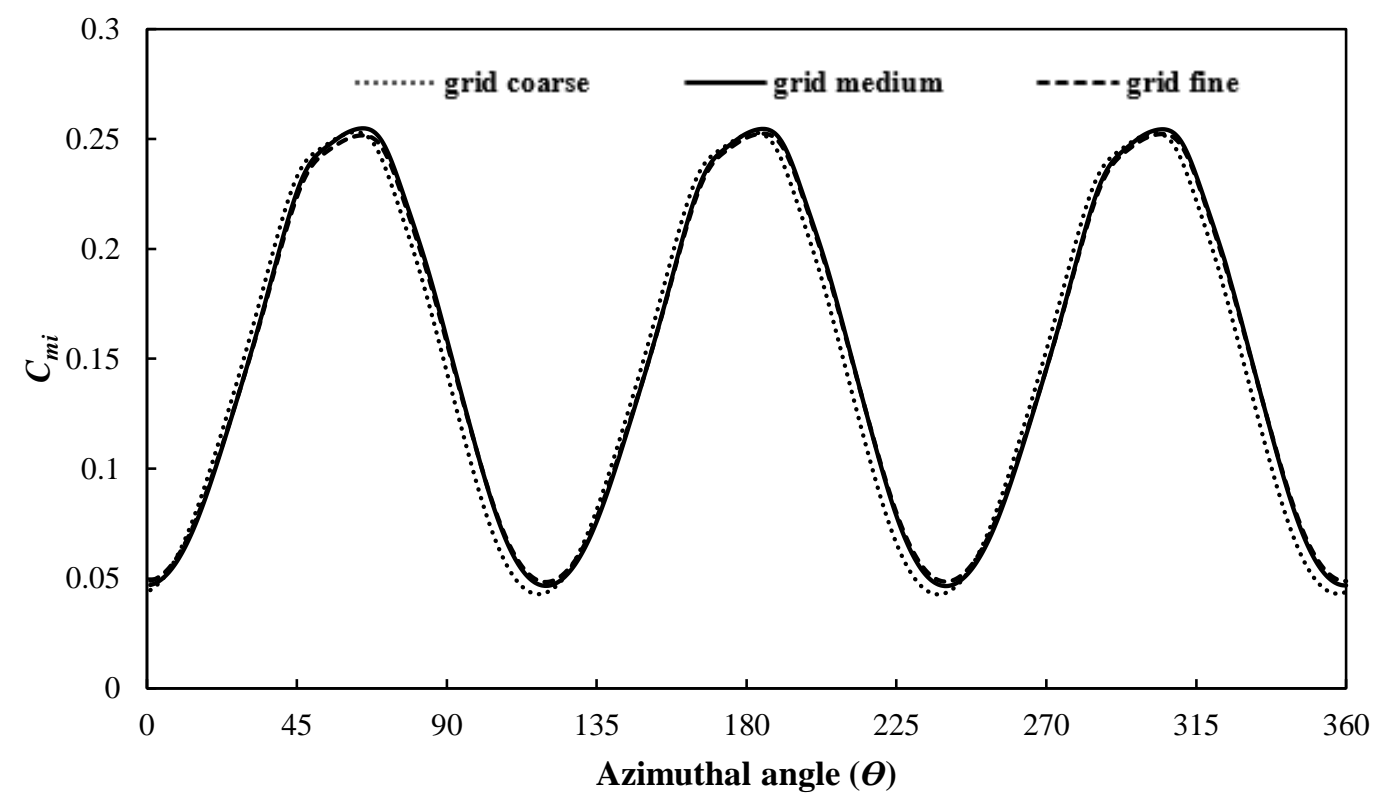

Figure 5. Comparison of instantaneous moment coefficients $C_{m i}$ of VAWT with different grids.

\subsection{Temporal resolution study}

As stated by McMullen et al. [38], the rate of rotation and the number of blades have major effect on the periodicity of relative motion of the rotating blades which is the main contributor to the unsteadiness of VAWTs. Castelli et al. [13] have considered this effect by defining a small time step equals to the lapse time of the rotor making a $1^{\circ}$ rotation. They also found that any further reduction of time step did not give better accuracy to the simulation results. To confirm their findings, the current simulation repeats the time independence study with three time steps setting corresponding to three time lapses for $1^{\circ}, 0.75^{\circ}$ and $0.25^{\circ}$ rotations, respectively. 


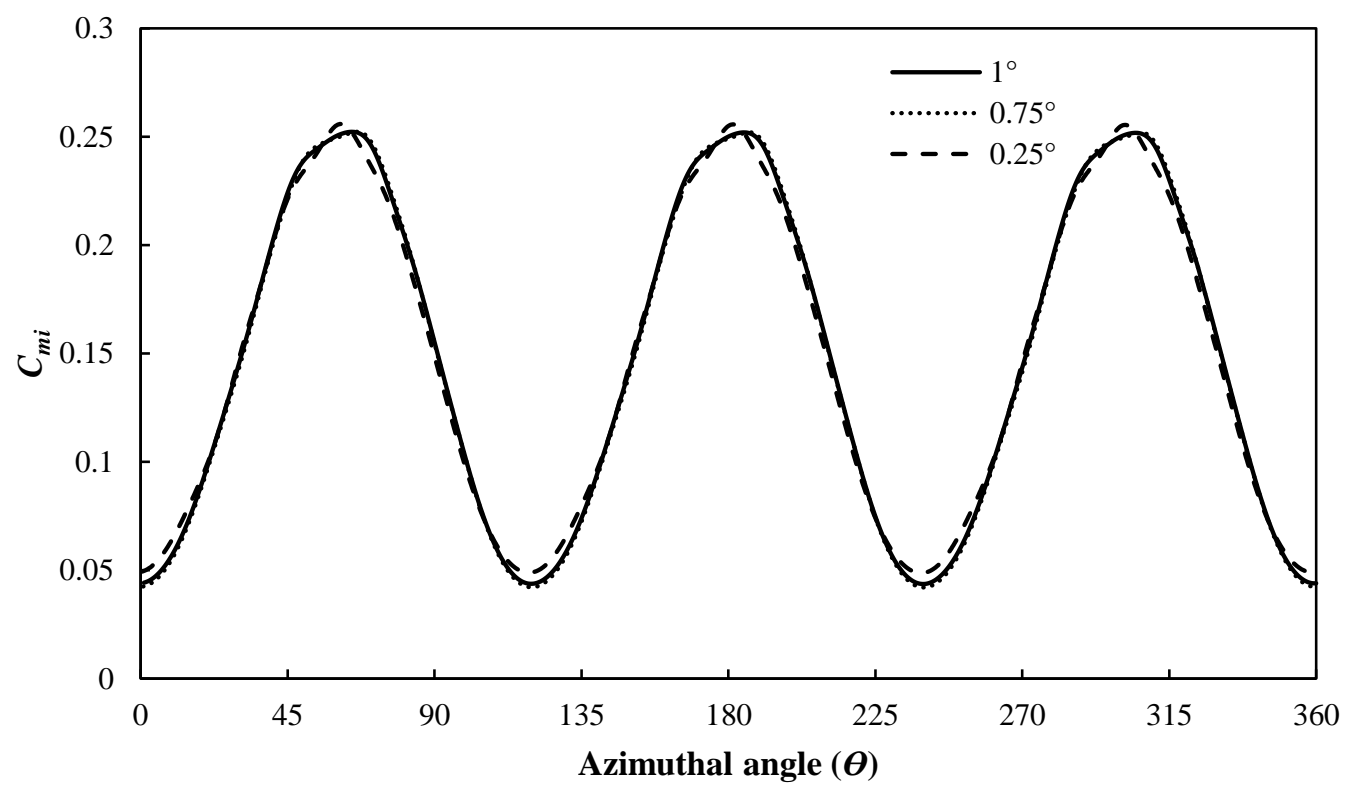

Figure 6. $C_{m i}$ distribution of three set of different time steps over one turbine revolution.

The instantaneous $C_{m}$ over one complete turbine revolution of three time steps in TSR = 2.5 are illustrated in Figure 6. It was found that there were no big differences on instantaneous $C_{m}$ between three time different steps. In addition, $C_{\text {pave }}$ between $1^{\circ}$ and $0.25^{\circ}$ time steps shows a small difference which is only $0.44 \%$. Hence the time step equal to the lapse of time of the rotor making a $1^{\circ}$ rotation is chosen for the rest of simulations.

\section{Impact of Turbulence Models}

\subsection{Revolution convergence analysis}

In VAWT simulation, it is important to obtain statistically converged flow field before collecting data samples. Hence, several studies have suggested some required number of turbine revolutions to run before collecting the data. It was usually defined by analysing the time history of moment coefficient $\left(C_{m}\right)$ or power coefficient $\left(C_{p}\right)$.

In previous URANS simulation, Castelli et al. [13] started the data sampling while the averaged $C_{m}$ over one turbine revolution $\left(C_{m a v e}\right)$ variations between two neighbouring revolutions is less than 1\%. Another study using Transition SST turbulence model [8] has found that the changes of $C_{\text {mave }}$ and $C_{\text {pave }}$ could be below $0.1 \%$ and $0.2 \%$ respectively after 20 revolutions, and the cumulated differences of these two values were as low as $1.06 \%$ and $2.41 \%$, respectively, between 20 and 100 revolutions. Therefore, using these examples, this study will start the data retrieval after the $C_{\text {mave }}$ variations fall under $0.1 \%$. 
In the case of URANS turbulence models, this study finds that the $C_{\text {mave }}$ dropped less than $0.1 \%$ compared to the previous revolution after 23 revolutions (see Figure 7) for both of RKE and SST turbulence models, confirming that a good convergence has been achieved. Hence, for the case of these two-equation URANS turbulence models, the data retrieval can be done at $24^{\text {th }}$ revolution. On the other hand, four-equation URANS turbulence model, i.e. Transition SST needs 27 revolutions before the $C_{\text {mave }}$ dropped less than $0.1 \%$. Therefore, the data can be collected at $28^{\text {th }}$ revolution for this turbulence model. TSST turbulence model takes longer to achieve statistically steady $C_{\text {mave }}$ than two-equation URANS turbulence models probably due to the addition of two more equations for the intermittency and momentum thickness Reynolds number.

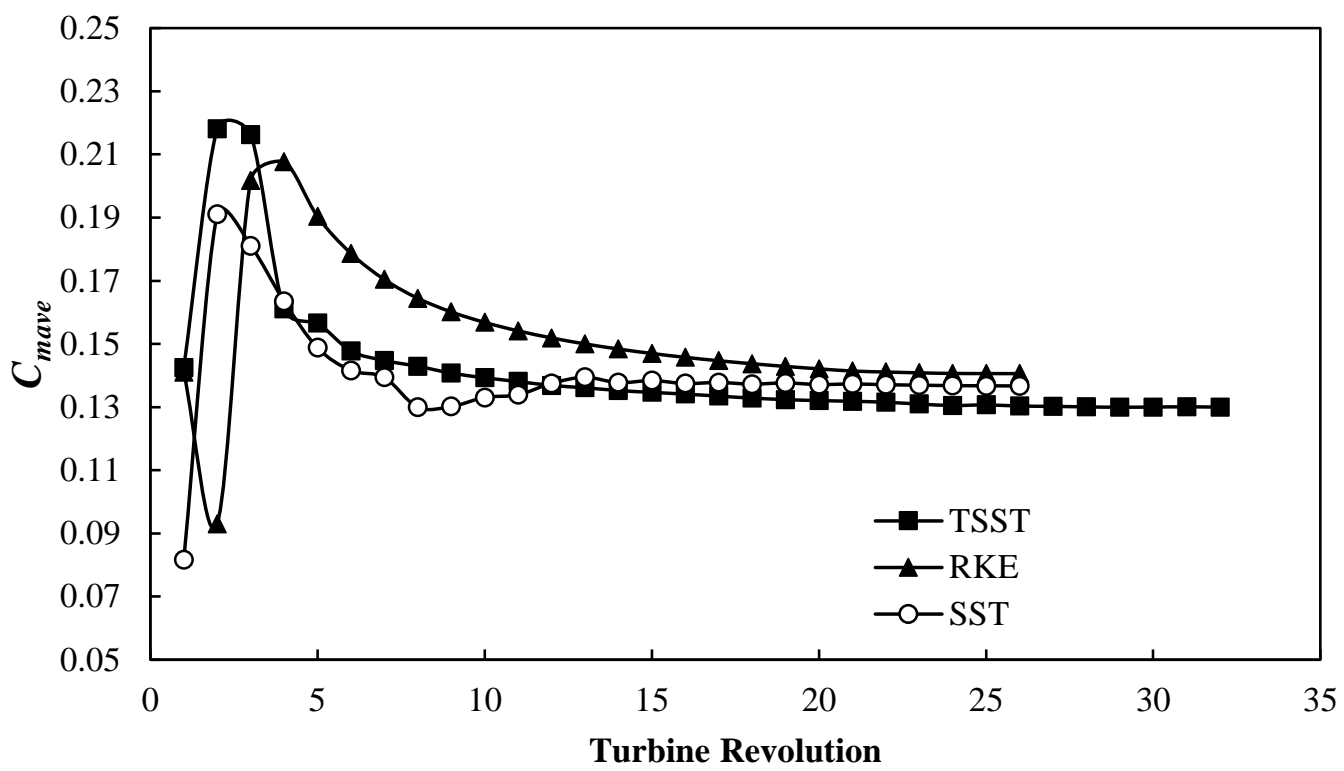

Figure 7. $C_{\text {mave }}$ changes over turbine revolution for URANS turbulence models.

For hybrid RANS-LES turbulence models, all four of the tested turbulence models need 34 revolutions before the $C_{\text {mave }}$ dropped less than $0.1 \%$ (see Figure 8 ). Compared to the URANS, hybrid RANS-LES turbulence models took more revolutions to reach convergence status. It is probably due to the fact that URANS turbulence models are mainly solving the mean flow and those large flow motions in the near field, and use ensemble averaging solution in the far field [39]. In contrast, hybrid RANS-LES turbulence model utilises the LES model in the far field, which can resolve the flow fluctuations to some extents and as a result, it will take longer time to achieve statistically converged flow field for both near and far fields. 


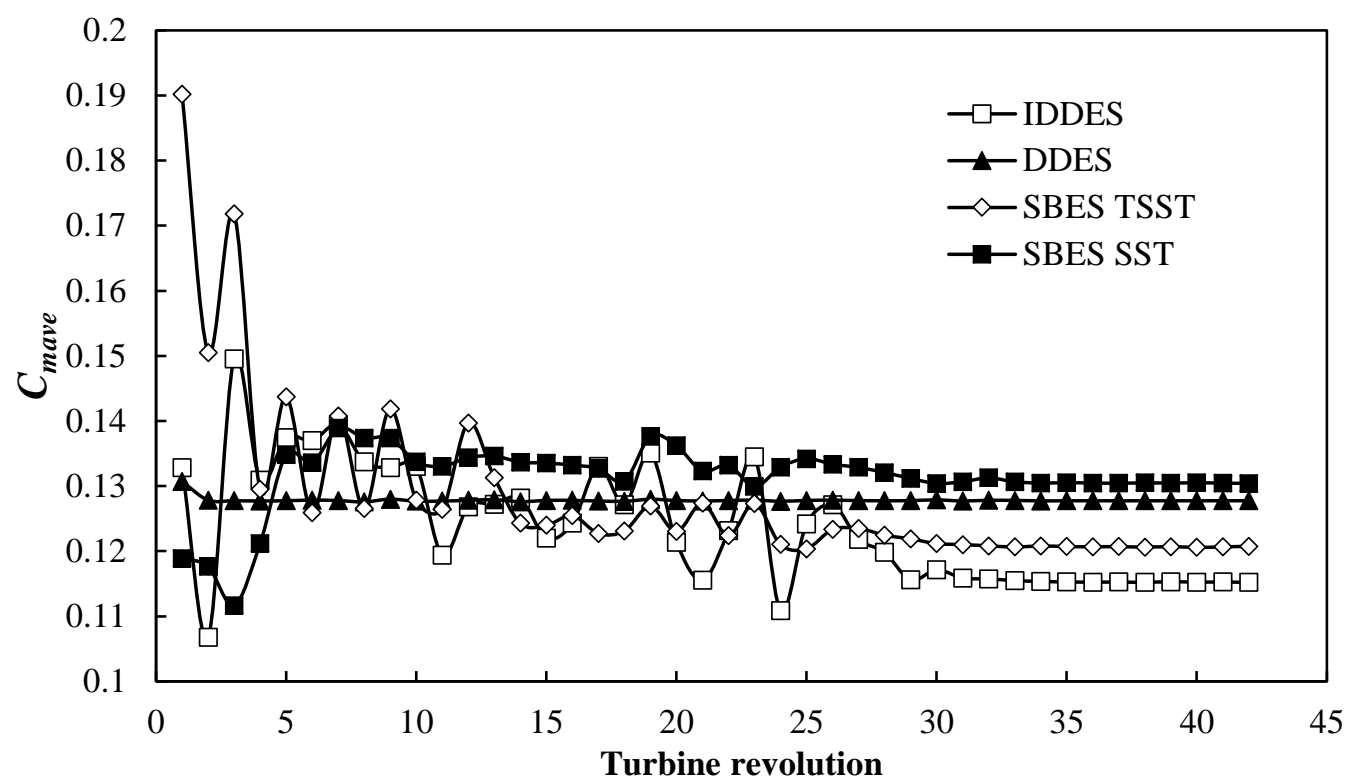

Figure 8. $C_{\text {mave }}$ changes over turbine revolution for hybrid RANS-LES turbulence models.

\subsection{Revolution averaged of turbine power coefficient $\left(C_{p a v e}\right)$}

\subsubsection{URANS turbulence models}

Figure 9 shows the revolution average $C_{p}$ prediction of current CFD simulation using twoequation URANS turbulence models (RKE and SST) and four-equation URANS turbulence model (i.e. TSST), compared with the experimental and the CFD simulation results of Castelli et al. [13] using RKE model. It is found the current CFD simulations can successfully generate the $C_{\text {pave }}$ curve in a similar trend as the experiment and CFD simulation results of previous study. It is also able to capture the optimum $T S R=2.64$, which gives the maximum $C_{\text {pave. }}$. In addition, current CFD gives better $C_{\text {pave }}$ prediction in all three TSR ranges than the CFD prediction of Castelli et al. [13], even for the same turbulence model (i.e. RKE). This might be due to the grid quality improvement, smaller time steps and higher order of residual convergence criterion and number of iterations. 


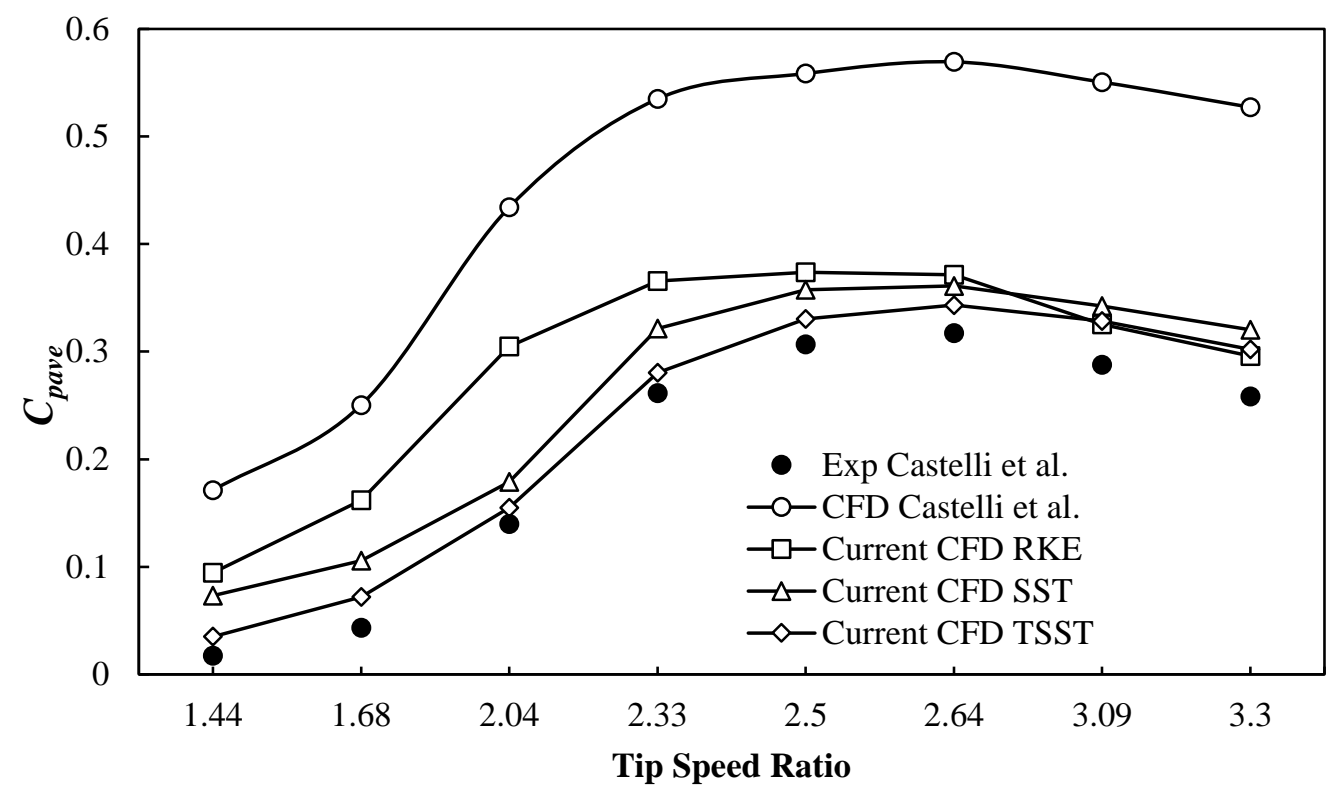

Figure 9. $C_{\text {pave }}$ comparison of current CFD simulation using RANS turbulence models and the experiment and CFD simulation using RKE results of Castelli et al. [13].

From Figure 10, it can be observed that two-equation URANS turbulence models (RKE and SST) still overestimate the experimental $C_{p}$ across the tested TSRs ranges. Compared to the medium and high ranges of TSRs, these turbulence models generate large discrepancy in low range of TSRs $(<2)$. This is understandable as flow around VAWTs will experience high level of unsteadiness at low TSRs range compared to medium and high ranges of TSRs $(>2)$. This behaviour is due to the fact that blades of VAWT are frequently exposed by higher absolute value of $A o A$ (the angle between the blade zero lift line and the free stream direction), even beyond the stall AoA, in low TSRs range compared to the medium and high ranges TSRs [40]. This condition can trigger dynamic stall with drag reduction called drag dynamic stall in high $A o A$. Moreover, flow around blades could experience enormous large viscous region in low TSRs range due to low Re number effects [10]. Hence, RKE generally would produce very large discrepancy as it has reputation to generate poor prediction accuracy of flow with prominent separation and over-prediction of turbulence kinetic energy. In addition, it also performs poorly in low Re number due to the need to generate viscous corrections simply to reproduce the law of the wall for flow with low Re number [23]. For SST, even though it still gives significantly large discrepancy, this turbulence model generates better prediction than RKE. As mentioned by Wilcox, $k-\omega$ model can significantly give more accurate prediction for flow with separation and with the absence of viscous correction, it can produce better prediction than $k$ - $\varepsilon$ model in flow with low Re number [23]. In cases of the medium and high ranges of 
TSRs, the flow around VAWTs relatively will be acquaintance with lower level unsteadiness as the blades will experience dynamic stall with lift increment in low $A o A$ similar to a sinusoidal pitching aerofoil [6]. Therefore, although the flow still has separation, it is relatively easier for these two turbulence models to generate more accurate prediction than in low TSRs range.

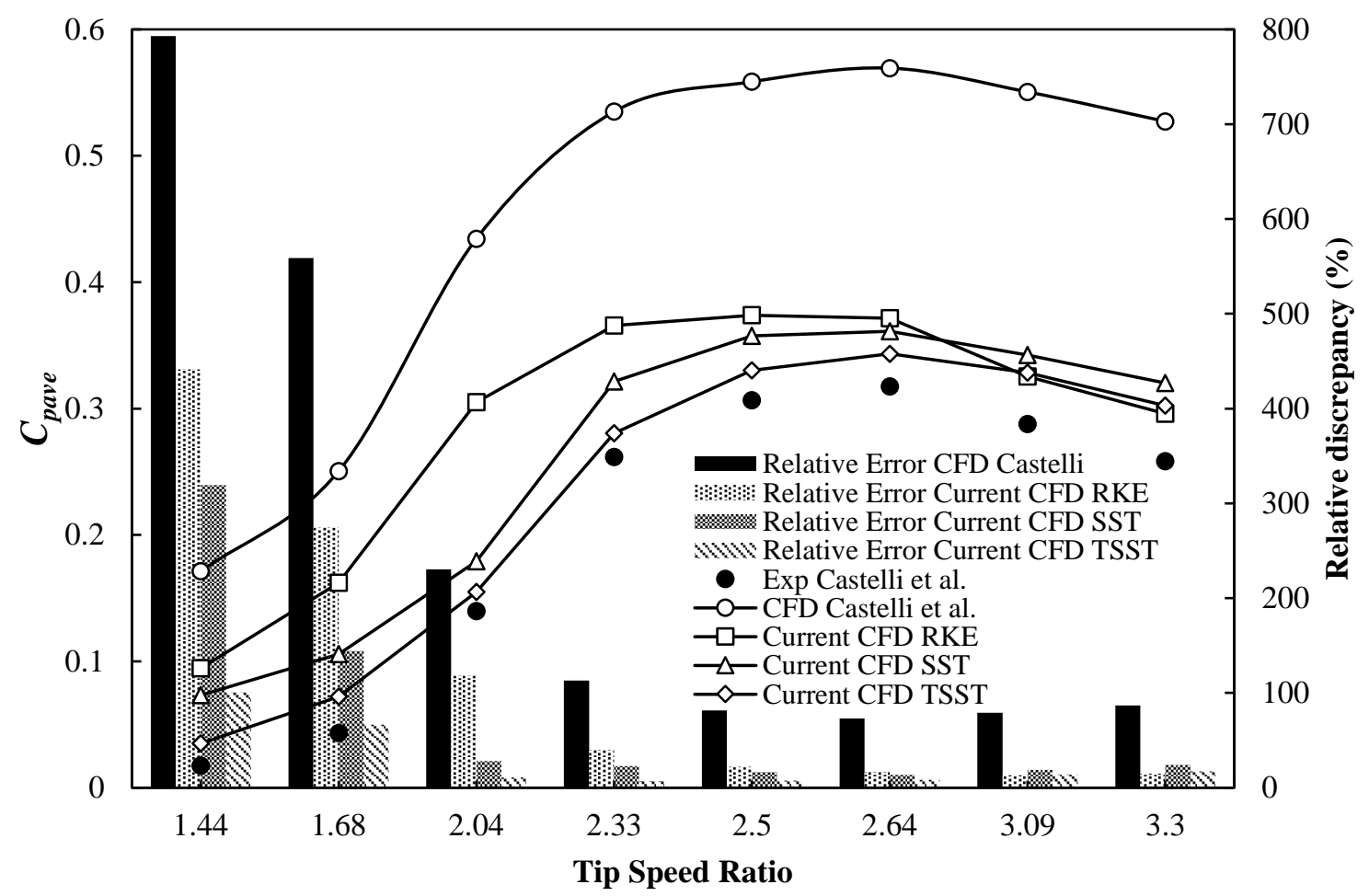

Figure 10. Comparison of $C_{\text {pave }}$ between current CFD simulation and the experiment and simulation of Castelli et al. [13], as well as relative discrepancies in percentage.

As mentioned above, RKE and SST turbulence models still give some considerable discrepancies in predicting $C_{\text {pave }}$ of VAWT. During the evaluation of the flow type around VAWTs, the flow is assumed to be predominantly laminar, as the estimated Reynolds number based on the free stream velocity and the blade chord, $R e_{U_{\infty}}$ is about 51000 . However, when the VAWT simulation is carried out using laminar viscous model, the results are far from the experiment and the instantaneous moment coefficient distribution. For this reason, it can be concluded that the flow does not retain the laminar flow characteristics. As the blades are rotating, the generated rotating speed will facilitate the development of flow turbulence. The Reynolds number based on the angular velocity $\left(R e_{\Omega}\right)$ (see Equation $(24)$ ) indicates that the flow is already in turbulence status even in the lowest $T S R$, i.e. $\operatorname{Re}_{\Omega}=449460$ in $T S R=1.44$, $\operatorname{Re}_{\Omega}=815760$, in $T S R=2.64$ and $\operatorname{Re}_{\Omega}=1019700$ in $T S R=3.3$, respectively. Note that the flow is categorised as turbulence flow if $\operatorname{Re}_{\Omega}$ is more than 60 , according to a previous study [41]. 


$$
R e_{\Omega}=\frac{\rho \omega_{t} R^{2}}{\mu}
$$

Hence, the blades of VAWT will experience laminar-to-turbulence flow transition from the incoming background flow to the zone of rotating flow [8].

The averaging process in equations of RKE and SST turbulence models does not actually accommodate the description of transitional flows where both of nonlinear and linear breakdown from laminar to turbulence effects are relevant [27]. The averaging method of these two models drops the effects of linear growth of the disturbance and as a result, there is a difficulty to apply these two models in the transition process [27]. To overcome, Transition SST turbulence model is applied for current CFD simulation. As described above, this model has two additional transport equations, one for $\gamma$ and the other for $\operatorname{Re}_{\theta}$, with the SST $k$ - $\omega$ transport equations. The two additional equations are applied to enable the SST $k$ - $\omega$ model to predict the transition onset and length [42]. Turbulence production limiters are also included in this model to eliminate an excessive turbulence kinetic energy prediction in the stagnation region. As illustrated in Figure 10, this model can significantly decrease discrepancy in all range of TSRs. These results suggest that the blades of VAWT indeed experience the transition flow.

\subsubsection{Hybrid RANS-LES turbulence models}

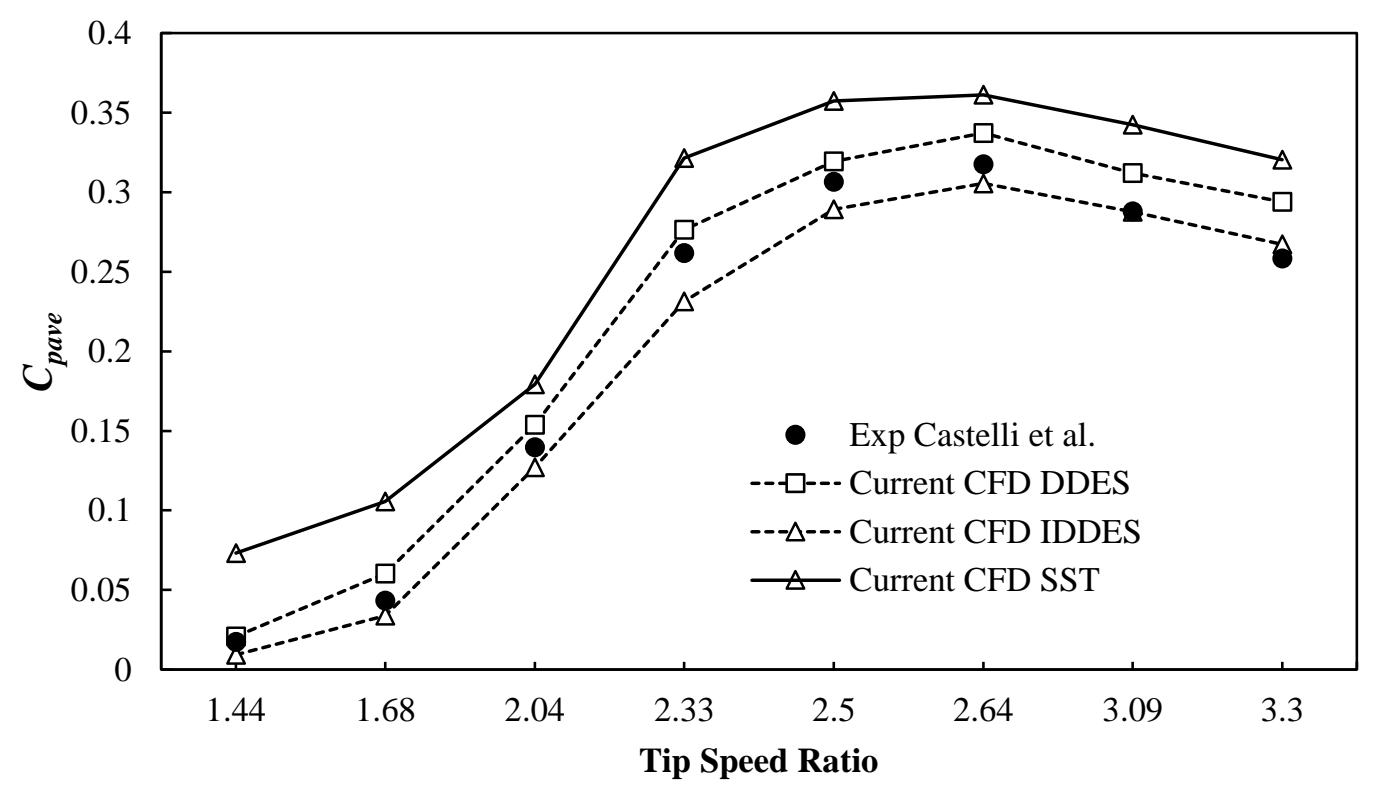

Figure 11. $C_{\text {pave }}$ comparison of current CFD simulation using DDES and IDDES turbulence models and the experiment results of Castelli et al. [13]. 
To further decreasing the discrepancy of CFD prediction, it is recognised that the use of LES or Direct Numerical Simulation (DNS) is preferable. However, the high demand of computing time and cost make these two methods hardly used in VAWTs CFD simulation. Hence, to reach the similar result of LES with less demand of computing time and simulation, hybrid RANS-LES turbulence models are preferred to be used in VAWTs CFD Simulation.

Figure 11 illustrates $C_{\text {pave }}$ prediction of current CFD simulation using hybrid RANS turbulence models called DDES and IDDES, compared with current CFD URANS SST $k-\omega$ simulation and the experiment results of Castelli et al. [13]. It is understood that these turbulence models can generate similar trend with the experiment results. Compared to SST, DDES can decrease the simulation discrepancy in all TSRs ranges (see Figure 12). It is rational as this turbulence model is based on SST model so it will inherit the ability of SST to give a good accuracy in flow with separation and low Re number [23]. In addition, the LES mode will decrease the discrepancy caused by RANS turbulence model. This mode can fix the problem of incorrect calculation of the momentum transport in the far field of RANS turbulence model (due to isotropic treatment of turbulence as a result of averaging method of RANS) by solving the large eddies in the far field using LES model. The incorrect calculation of momentum transport in the far field can generate an unwanted greater intermittency [43] (For example, turbulence heat fluxes caused by RANS simplification have basically no effect on the mean temperature. While in fact, the turbulence fluctuations may produce significant change of properties in space and instantaneous properties in time [44]). For IDDES, while all of URANS turbulence models and DDES overestimate the $C_{\text {pave }}$ in all range of TSRs, this model underestimate all of $C_{\text {pave }}$ except for the prediction in $T S R=3.3$. Nonetheless, this model can generate relatively smaller discrepancy than DDES (in comparison, IDDES generates average discrepancy across all ranges TSRs around 9\% while DDES gives about 13.5\%).

Despite of the ability to produce better results, these two models (DDES and IDDES) have some complexities during the grid generation. Same as other standard DES methods, DDES and IDDES have the grid requirement of LES method in the far field that can still add a great amount of computational time and cost. Moreover, due to the use of zonal method by changing from RANS to LES mode, DES is very sensitive to the local grid-size, mostly in the grey area where the model changes from RANS to LES mode which can introduce problem called modelled-stress depletion (MSD). It occurs because when the grid is gradually refined or the geometry features demand a fine wall-parallel grid or a boundary layer thickens and near flow separation (leading to ambiguous grid), the modelled Reynolds stress will solve "unreal" stress to restore the balance due to eddy viscosity reduction caused by DES limiter activation. MSD 
can lead to "unreal" separation called GIS [31]. Even though this problem has been fixed in DDES and IDDES, it still can appear in extreme grids and separations which VAWT model usually has.

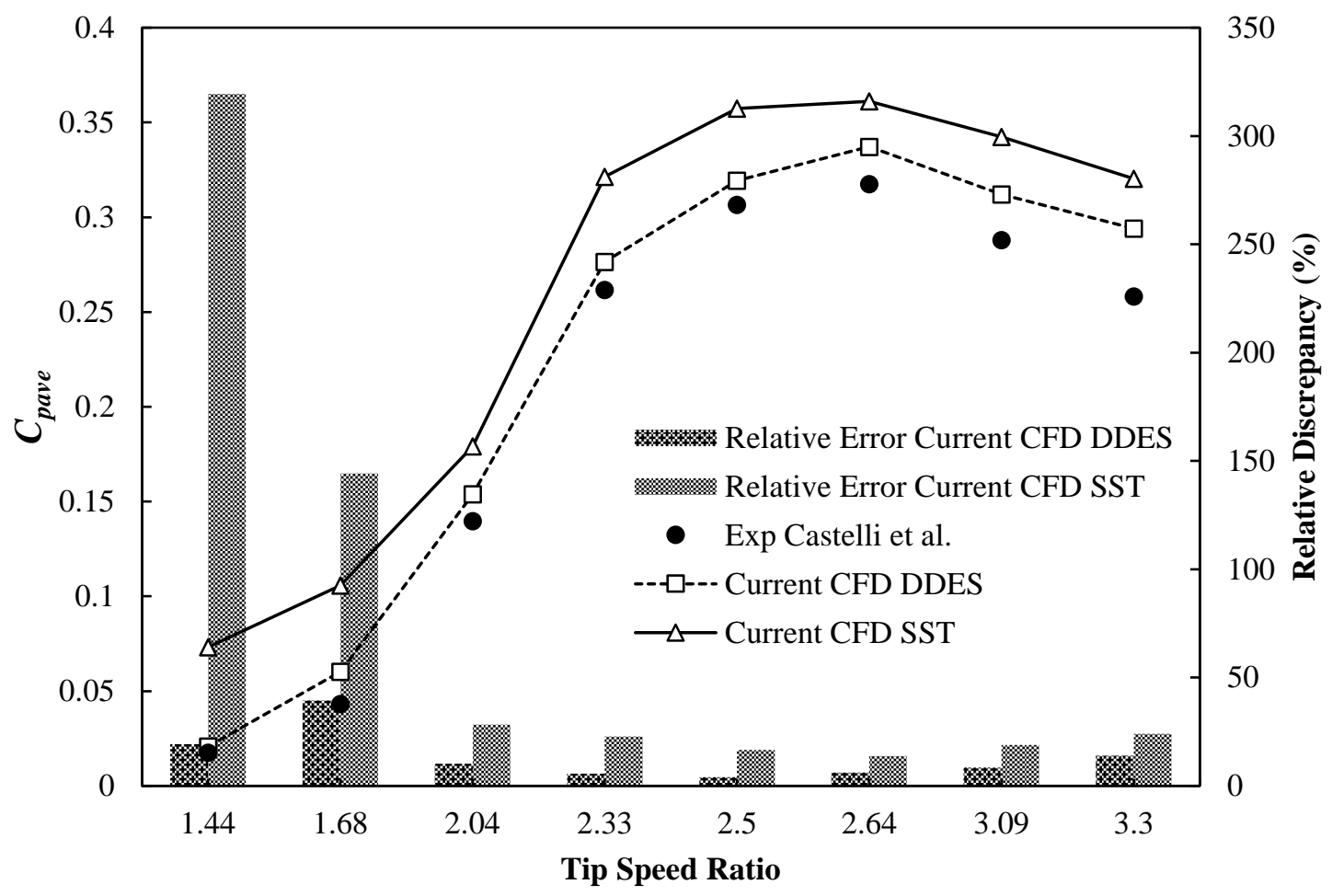

Figure 12. Comparison of $C_{\text {pave }}$ between current DDES and Transition SST CFD simulation and the experiment of Castelli et al. [13], as well as relative discrepancies in percentage.

To overcome the grid development difficulty, a relatively new hybrid RANS-LES model called SBES is applied in the current CFD simulation [14]. As mentioned above, this turbulence model allows a RANS-LES “transition” even on a coarser grid that other DES models cannot. Based on Figure 13, it can be observed that, SBES turbulence model can reproduce the trend line of experiment results and generate relatively similar results with DDES and IDDES for both SST $k-\omega$ and Transition SST based SBES turbulence models. Moreover, SBES Transition SST turbulence model performs better than its SST $k-\omega$ version in all TSRs range except for the $T S R=1.44$ (see Figure 14). It might be due to the fact that the flow in this TSR has very low Re number and the transition behaviour is not as strong as in higher TSRs. Hence, SST $k$ $\omega$ version of SBES performs better than its Transition SST version. In addition, compared to Transition SST turbulence model (the best model for URANS model in current CFD simulation), the SBES SST $k$ - $\omega$ produces larger discrepancy in $T S R \geq 2.64$ (see Figure 14). This again might be due to the fact that transition flow becomes stronger in higher TSRs compared to lower TSRs and therefore Transition SST turbulence model is more suitable than 
SBES SST $k-\omega$. Overall, SBES Transition SST turbulence model generates smallest discrepancy (e.g., on average, discrepancy across all ranges of TSRs is around 8.9\%) compared to all of RANS and hybrid RANS-LES turbulence models.

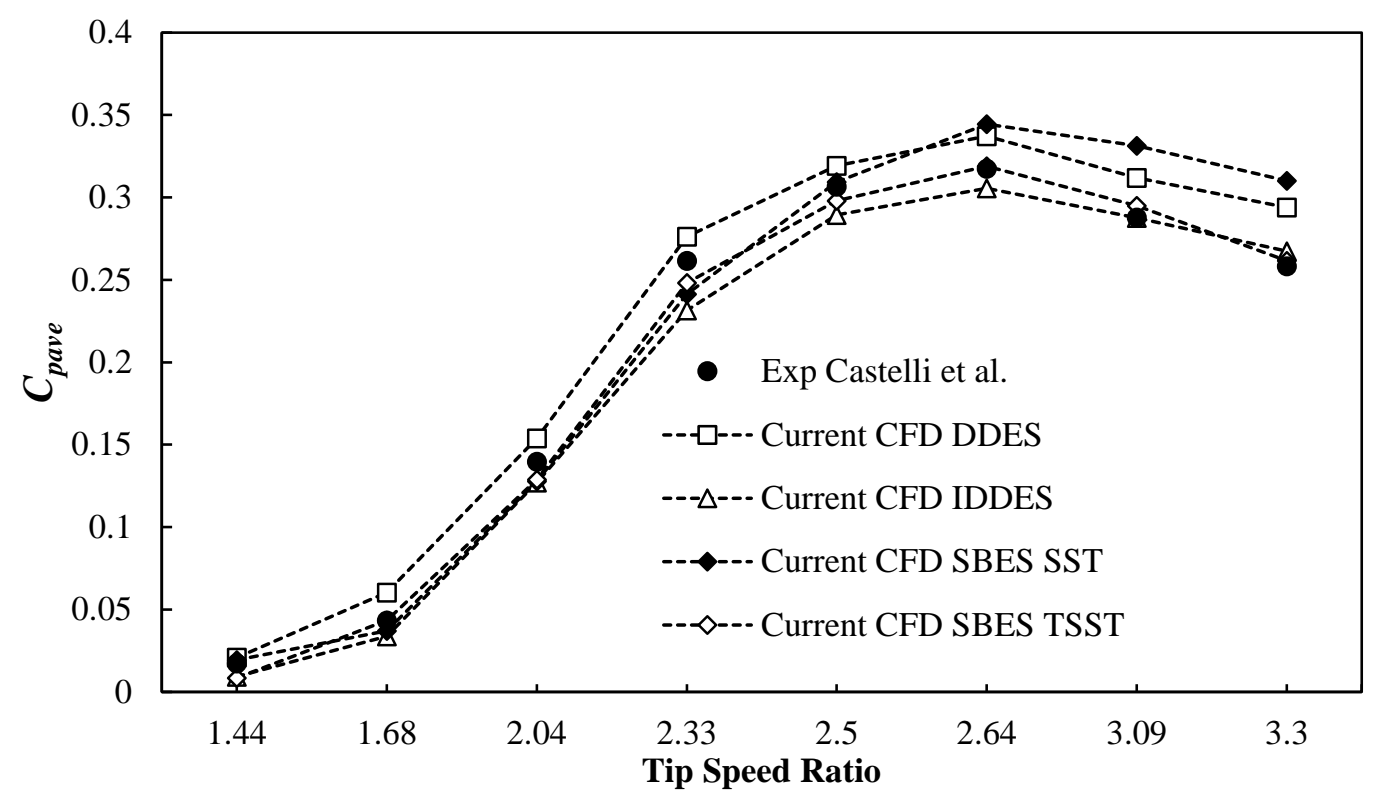

Figure 13. $C_{\text {pave }}$ comparison of current CFD simulation using SBES turbulence models based on SST $k-\omega$ and Transition SST RANS turbulence models and the experiment results of Castelli et al. [13].

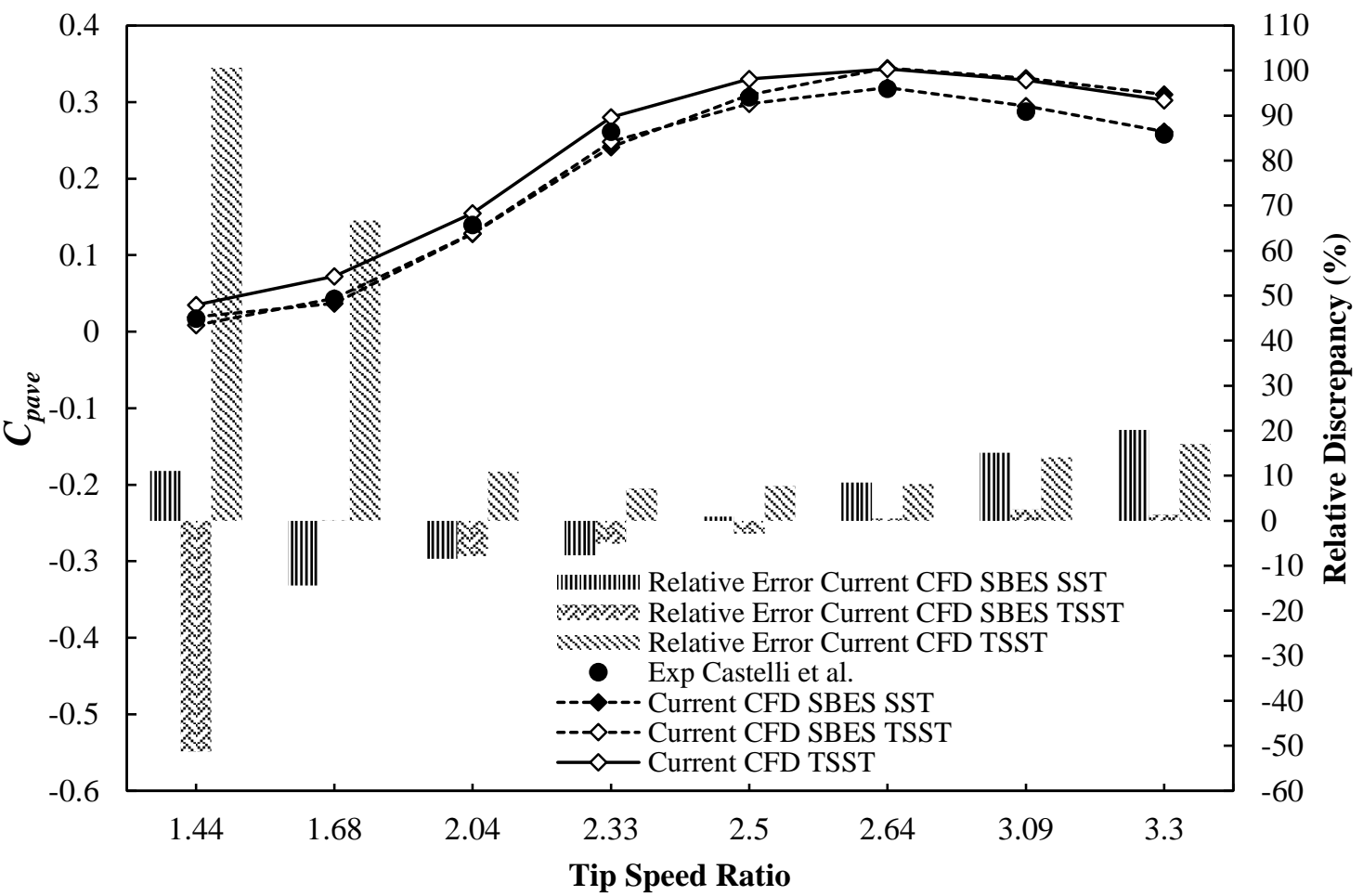

Figure 14. Comparison of $C_{\text {pave }}$ between current SBES and Transition SST CFD simulation and the experiment of Castelli et al. [13], as well as relative discrepancies in percentage. 
It can be observed that while the use of appropriate turbulence model can improve the CFD predictions to some extents, simulation results still exhibit some differences compared to experimental results at all ranges of TSRs. It is likely due to the fact that the experimental measurements of Castelli et al. [13] are 3D data. As mentioned above (see Section 2), this difference is mainly caused by the combined effects of finite blade length and spokes drag.

It is worth noting that the primarily purpose of this $2 \mathrm{D}$ simulation study is to compare various turbulence models and assess their capability in predicting the power coefficient of VAWT quantitatively and visualising the flow around VAWT qualitatively. While a full 3D simulation is preferable to capture flow separation, previous study using a hybrid LES-RANS model (DES) showed that 2D simulation could produce quite similar results as 3D simulation on terms of turbulent flow separation on the blade [45].

\subsection{Turbine aerodynamics characteristics over a revolution}

\subsubsection{URANS turbulence models}

The detailed comparison of $C_{\text {pave }}$ prediction of three different URANS turbulence models already showed that while TSST model was superior in low to medium ranges of TSRs, its performance at high ranges of TSRs was mostly the same as RKE and SST models. Hence,

further investigation to understanding these differences is discussed in each range of TSRs in this section.

\section{1) Low range of TSR (Representative TSR $=1.44$ )}

Figure 15 displays the prediction of instantaneous moment coefficient $C_{m i}$, i.e. $C_{m}$ value in every time step $\left(1^{\circ}\right)$, distribution of blade 1 during one revolution $\left(360^{\circ}\right.$ rotation). It shows that SST and TSST models produce lower maximum $C_{m i}$ and earlier greater first fall of $C_{m i}$ (around azimuthal angle $(\theta)=57^{\circ}$ ) indicating these two models predict earlier and stronger flow separation compared to RKE. It can be also seen that in all three models, $C_{m i}$ distribution is always fluctuating with greater and similar magnitude predicted by SST and TSST models. This fluctuation demonstrates that there is a strong flow separation almost in all azimuthal angles when VAWT is operated in low TSRs range due to the blade is mostly operated in stall condition. Furthermore, even though SST and TSST models give similar prediction, overall TSST model predicts lower value of $C_{m i}$ resulting in lower value of $C_{p}$. Hence, TSST model can give smallest discrepancy of $C_{\text {pave }}$ prediction in this TSR. 


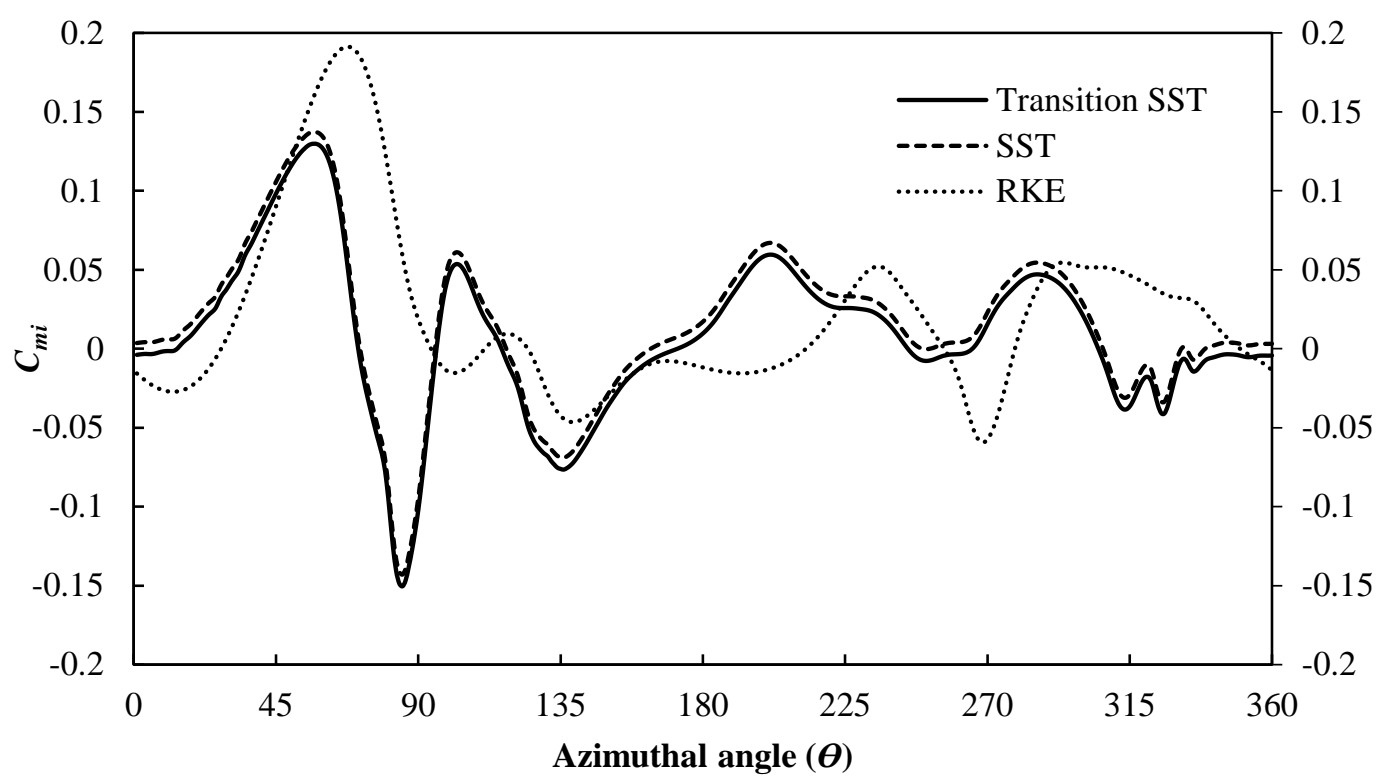

Figure 15. Comparison of instantaneous moment coefficient $\left(C_{m i}\right)$ distribution of three URANS models (RKE, SST and TSST) in TSR $=1.44$. 


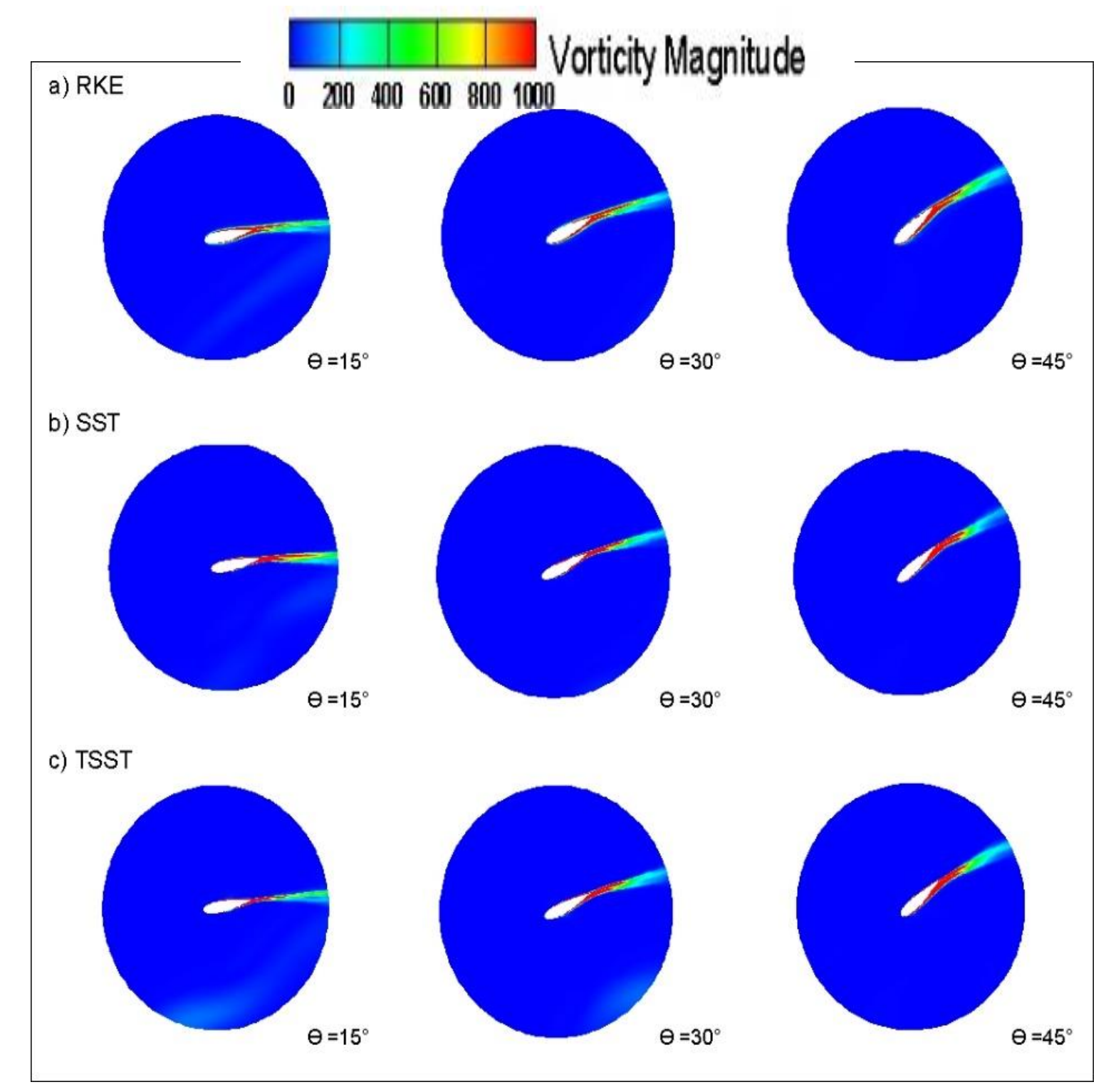

(a) 
a) RKE

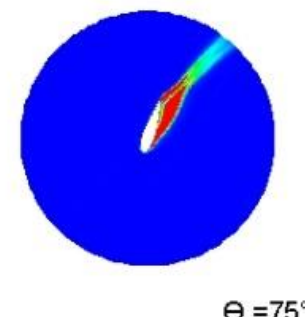

$\Theta=75^{\circ}$

b) SST

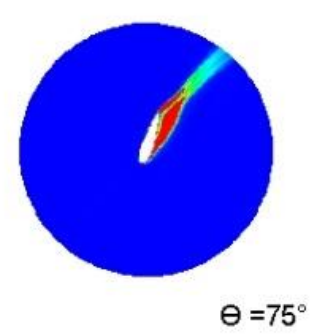

c) TSST

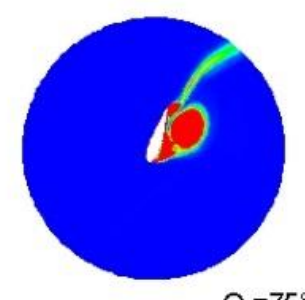

$\theta=75^{\circ}$

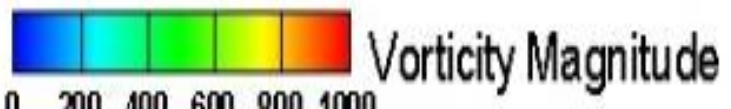

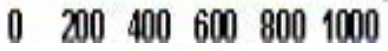

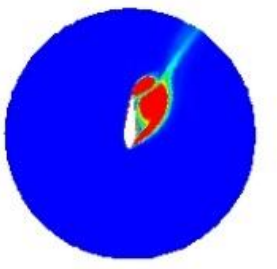

$\Theta=90^{\circ}$

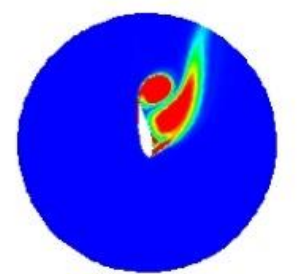

$\theta=105^{\circ}$

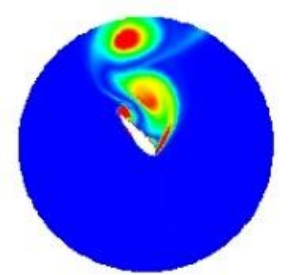

$\theta=135^{\circ}$

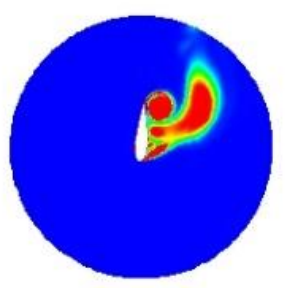

$\ominus=90^{\circ}$

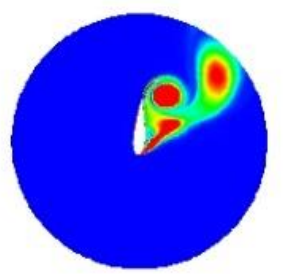

$\ominus=90^{\circ}$

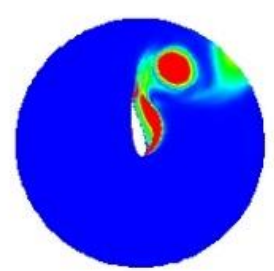

$\Theta=105^{\circ}$

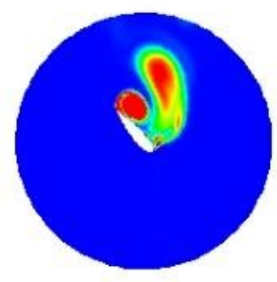

$\theta=135^{\circ}$

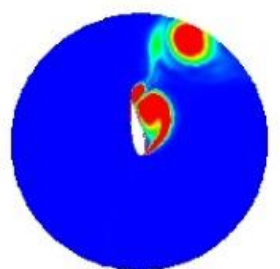

$\theta=105^{\circ}$

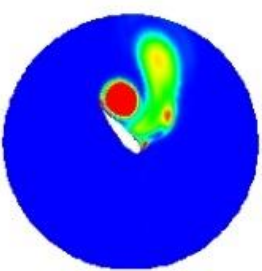

$\theta=135^{\circ}$

(b)

Figure 16. Comparison of contour plots of $z$-vorticity $(\xi)$ indicating the process of flow separation at important azimuthal positions in TSR $=1.44$ (URANS models), (a) before vortex shedding and (b) after vortex shedding.

To confirm those aforementioned behaviour, Figure 16 illustrates the contour plots of $z$ vorticity at several important azimuthal angles of blade 1 to understand the prediction of unsteady separation process of each model. $z$-vorticity is calculated by taking the curl of velocity vector $(\vec{U})$ (see Equation $(25))$.

$\xi=\nabla \times \vec{U}=\frac{\partial U_{y}}{\partial x}-\frac{\partial U_{x}}{\partial y}$

where $U_{y}$ is $y$-velocity and $U_{x}$ is $x$-velocity. 
It can be seen that from $\theta=0^{\circ}$ to $45^{\circ}$, there is a development of trailing and leading edges vortices with three URANS models predict similar growth behaviour of these vortices (see Figure 16a). However, when the flow starts to experience dynamic stall (the vortex shedding starts at a location around $\theta=50^{\circ}$, for $R e_{\Omega}=61800$ ), RKE and SST models predict a later strong vortex shedding development on leading edge with no flow vortex shedding in trailing edge vortex compared to TSST. As shown in Figure 16b, at $\theta=75^{\circ}$, TSST already predicts that there are strong leading and trailing edges vortices shedding leading to development of dynamic stall and roll up trailing edge vortices. In addition, as the degree of azimuthal angle increases, SST and TSST give similar vortex development with larger vortex shedding far from the wall compared to RKE.

2) Medium range of TSR (Representative TSR $=2.64)$

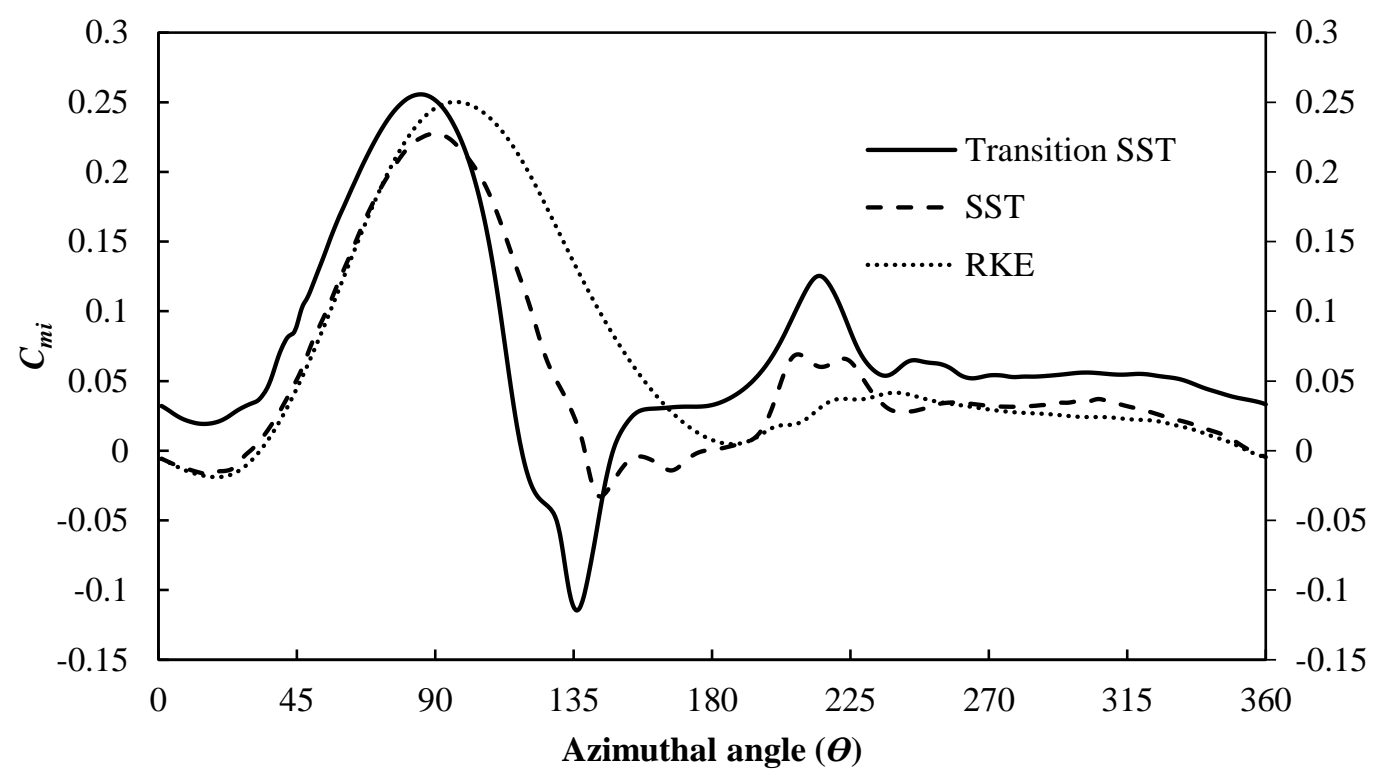

Figure 17. Comparison of instantaneous moment coefficient $\left(C_{m i}\right)$ distribution of three URANS models (RKE, SST and TSST) in TSR $=2.64$.

For optimum $T S R, C_{m i}$ distribution shows a smaller fluctuation indicating that flow separation strength is reduced at this TSR due to smaller position range where blade experiences dynamic stall. As illustrated in Figure 17, TSST model predicts earlier and steeper fall of $C_{m i}$ $\left(\theta=90^{\circ}\right)$ indicating that this model predicts earlier and stronger separation compared to other URANS models in the optimum value of TSR. Figure 17 also demonstrates that RKE model does not generate negative value during the fall of $C_{m i}$ showing that this model does not predict strong separation unlike SST and TSST models. In addition, from $\theta=135^{\circ}$ to $260^{\circ}$ where dynamic stall occurred, TSST and SST models produce fluctuation of $C_{m i}$ suggesting the 
process of vortex shedding in these azimuthal angles. As TSST model generate higher drop of $C_{m i}$ during dynamic stall, this model predicts lower power generation compared to other URANS models and as a result, it gives a closer $C_{\text {pave }}$ value to the experiment result.

It is important to understand the flow behaviour associated with the results above. Figure 18a shows that there is a development of trailing and leading edges vortices with three URANS models predict similar behaviour of these vortices growth from $\theta=0^{\circ}$ to $90^{\circ}$. This result is identical in lower TSR which means that RKE model can generate similar prediction with SST and TSSTs model when the dynamic stall is still not occurred (the vortex shedding starts at a location around $\theta=95^{\circ}$, for $\operatorname{Re}_{\Omega}=215270$ ). At $\theta=135^{\circ}$ (when the dynamic stall is already occurred and vortex shedding appears), RKE model only suggests that there is a small vortex shedding in trailing edge generating secondary trailing edge vortices without leading edge vortex shedding (see Figure 18b). On the other hand, SST and TSST models predict a strong vortex shedding in both trailing and leading edges indicating the present of dynamic stall, trailing edge roll up and secondary vortices which match with earlier work [8]. These vortex shedding are associated with the fluctuation of $C_{m i}$ in the region where dynamic stall occurred. Note that the TSST model generates stronger shedding compared to SST model. Hence, TSST model produces a larger fluctuation of $C_{m i}$ as illustrated in Figure 17. 


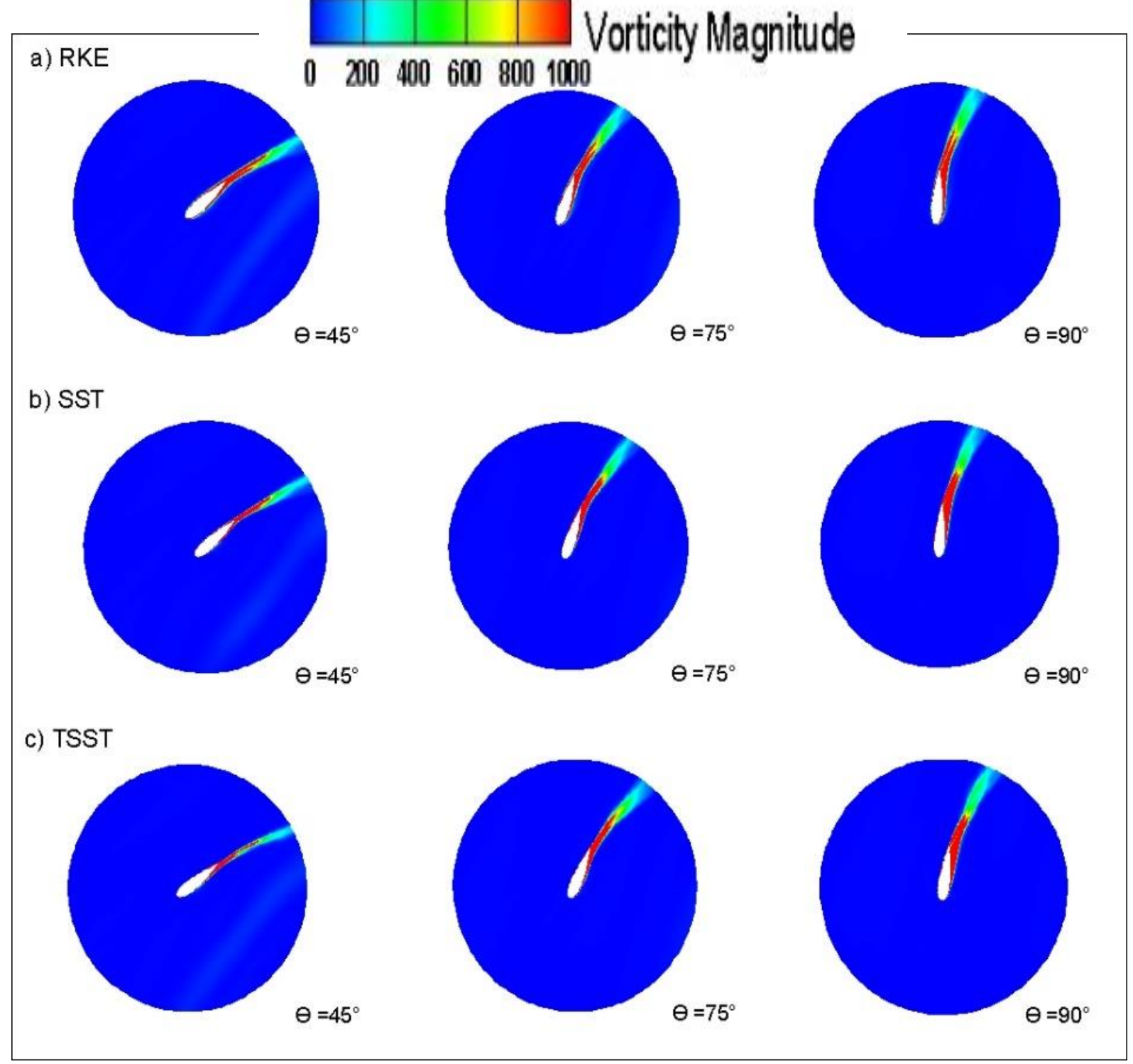

(a) 
a) RKE

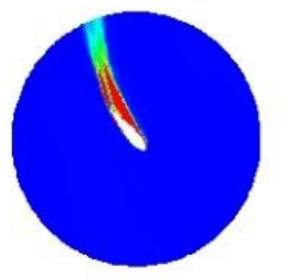

$\theta=135^{\circ}$

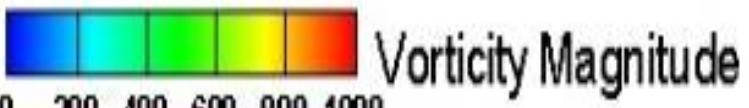

$0 \quad 2004006008001000$
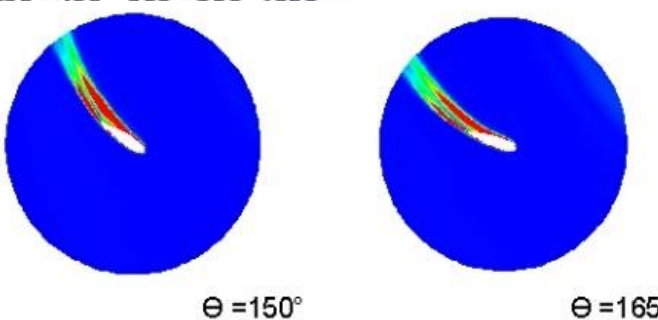

$\theta=165^{\circ}$

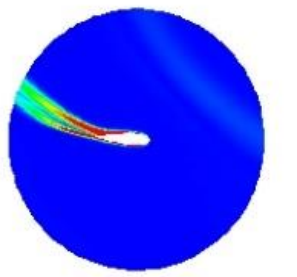

$\theta=180^{\circ}$

b) SST
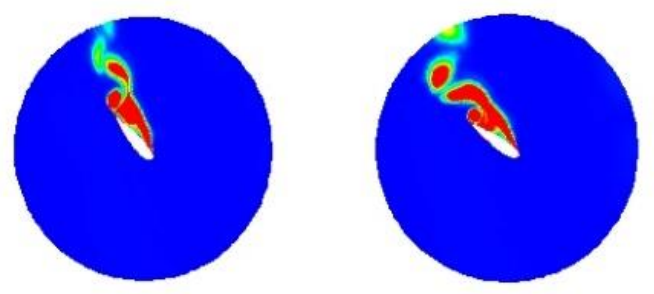

$\theta=150^{\circ}$
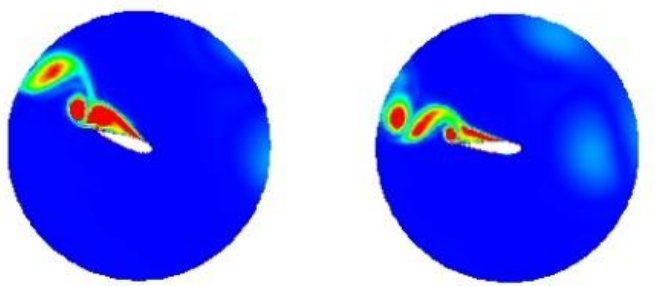

$\theta=135^{\circ}$

$\Theta=180^{\circ}$

c) TSST
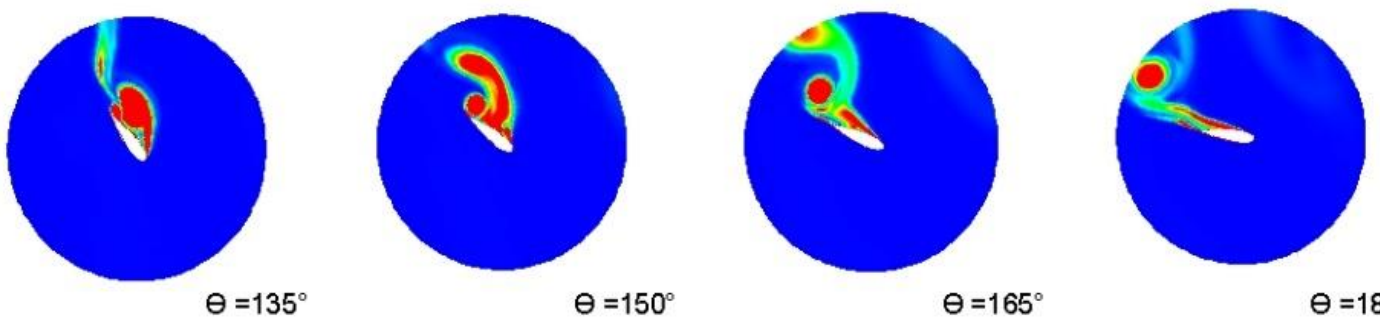

$\theta=180^{\circ}$

(b)

Figure 18. Comparison of contour plots of $z$-vorticity $(\xi)$ indicating the process of flow separation at important azimuthal positions in TSR $=2.64$ (URANS models), (a) before vortex shedding and (b) after vortex shedding.

\section{3) High range of TSR (Representative TSR $=3.3$ )}

As mentioned above, VAWTs experience a smaller range of angles of attack in high TSRs range compared to low and medium ranges of TSRs. Even though the turbine operates in the ranges of no static stall condition, the power production will decrease due to the higher rotation of the turbine compared to incoming wind with the addition of high vibration and drag and tip losses [46]. The loss in power production is confirmed by the optimum value of $C_{m i}$ prediction of all three URANS models falling under optimum TSR. It is noted that all three URANS models produce similar trend line of $C_{m i}$ with small difference over one turbine revolution (see Figure 19). Nevertheless, TSST model predicts the lowest range values of $C_{m i}$ and earlier fall 
of $C_{m i}$ compared to RKE and SST models. Hence, TSST still gives the lowest value of $C_{p}$ prediction although it is still quite similar with RKE and SST predictions. It also can be observed that these URANS models predict that the $C_{m i}$ distribution does not have a great fluctuation, indicating that there is no strong vortex shedding at high range of TSRs. This phenomenon can be verified by Figure 20. As illustrated, in this TSR, after developing trailing and leading edge vortices from $\theta=0^{\circ}$ to $90^{\circ}$, there is only a small vortex at the trailing edge where the vortex is not detached from the blade (the vortex shedding starts at a location of around $\theta=110^{\circ}$, for $\operatorname{Re}_{\Omega}=311575$ ). This shows that in high TSRs range, VAWT loss in power production is not mainly because of dynamic stall but by the fact that the rotor can act as an obstructed solid wall due to high rotation speed [47]. It is worth to mention that, all URANS models also predict similar vortex growth at this TSR regime resulting similar prediction in power generation.

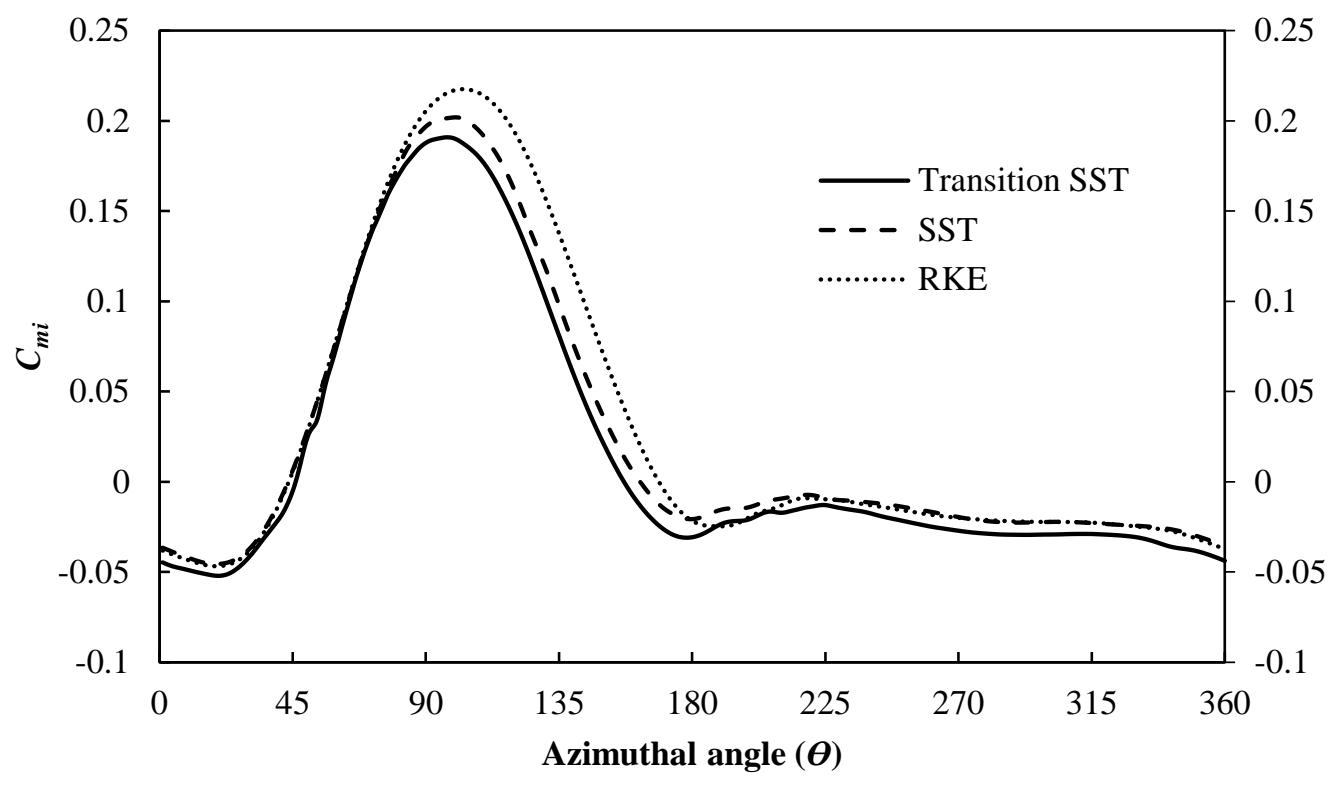

Figure 19. Comparison of instantaneous moment coefficient $\left(C_{m i}\right)$ distribution of three URANS models (RKE, SST and TSST) in TSR $=3.3$. 


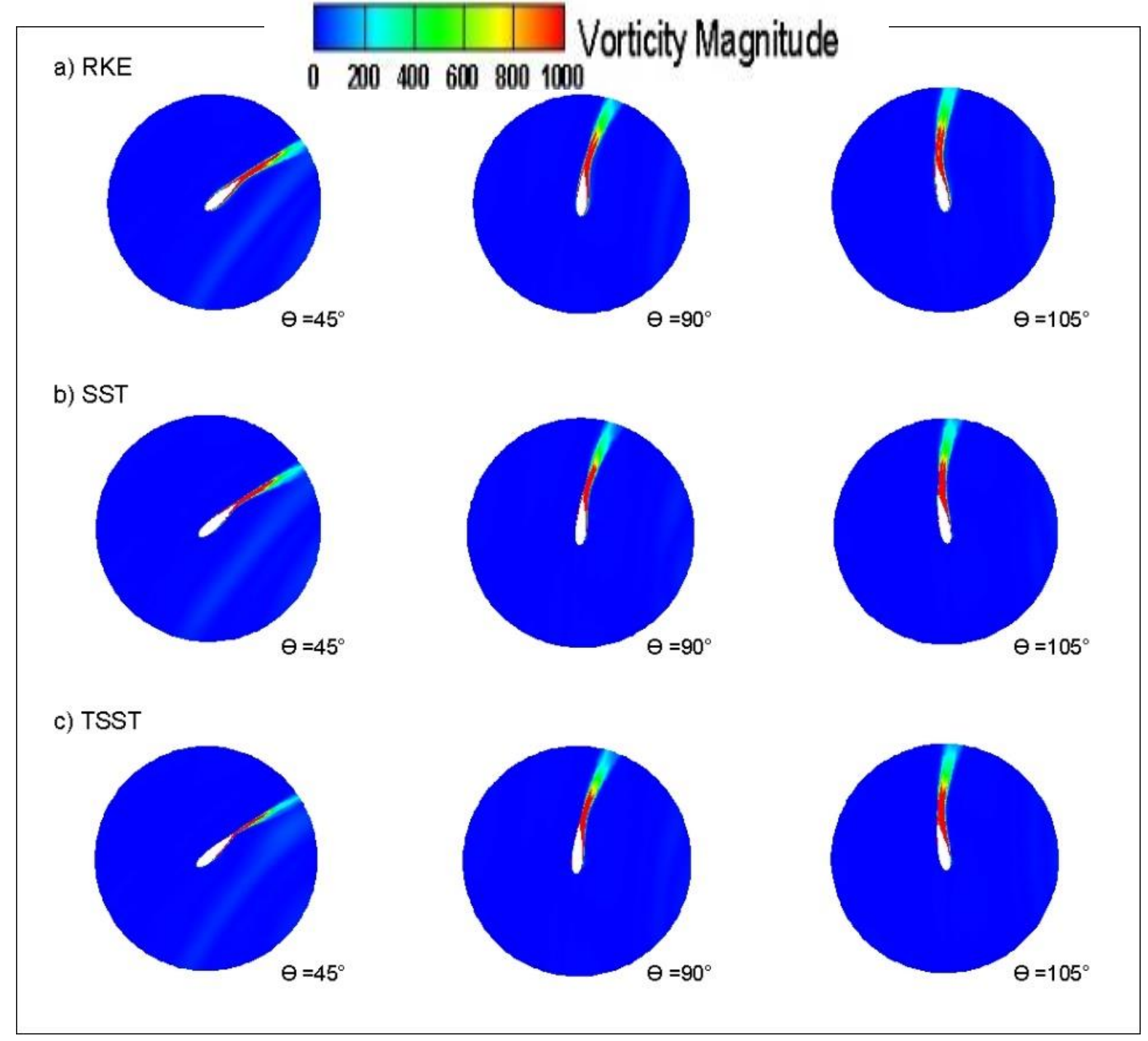

(a) 
a) RKE

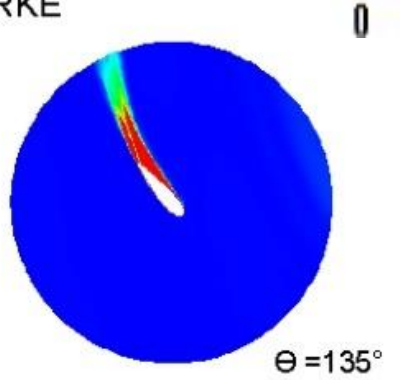

\section{Vorticity Magnitude}

2004006008001000
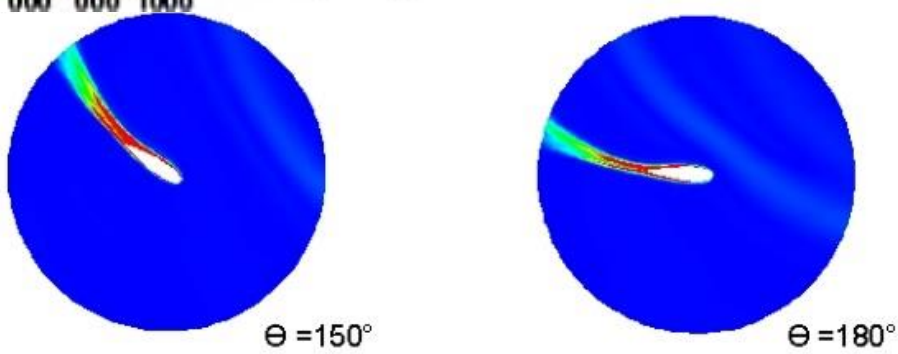

b) SST
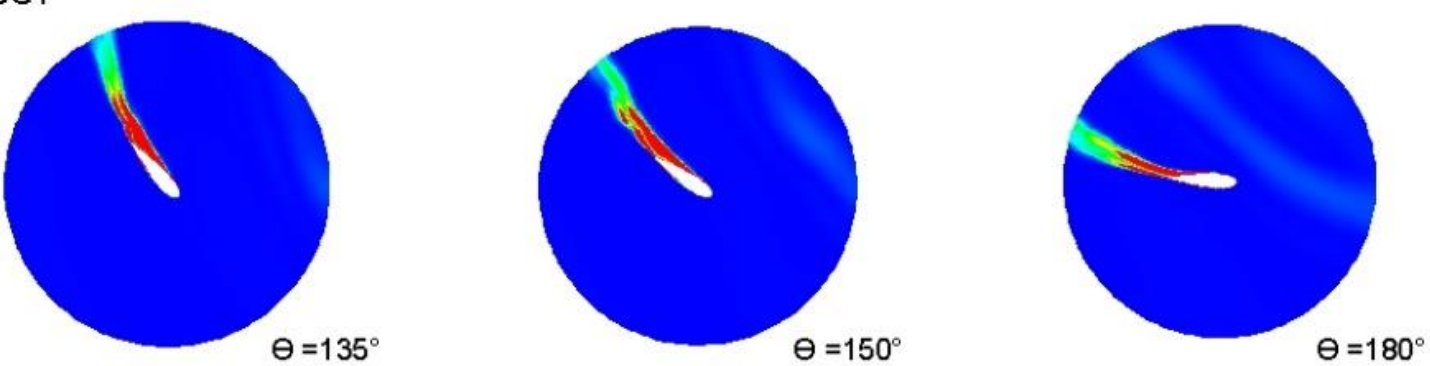

c) TSST
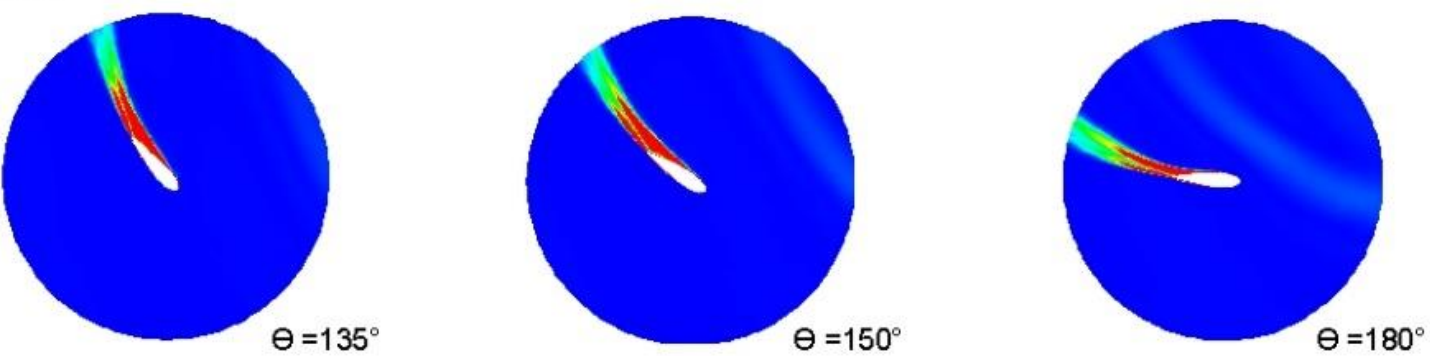

(b)

Figure 20. Comparison of contour plots of $z$-vorticity $(\xi)$ indicating the process of flow separation at important azimuthal positions in TSR $=3.3$ (URANS models), (a) before shedding and (b) after shedding.

\subsubsection{Hybrid RANS-LES Turbulence Models}

The detailed comparison of $C_{\text {pave }}$ prediction of four different hybrid RANS-LES turbulence models shows that they can reduce the inaccurate prediction of URANS turbulence models in all ranges of TSRs. Therefore, further investigation to understand these differences is discussed in each TSRs range in this section. 


\section{1) Low range of TSR (Representative TSR $=1.44)$}

Figure 21 presents the prediction of $C_{m i}$ distribution of four hybrid RANS-LES turbulence models in TSR $=1.44$. It is noticed that SBES TSST predicts earliest and steepest fall of $C_{m i}$ value compared to other hybrid RANS-LES models. This indicates that this model predicts earlier start of dynamic stall. Furthermore, it also generates lowest peak value of $C_{m i}$. Hence, this model predicts the lowest value of $C_{\text {pave }}$ than URANS models and other hybrid RANSLES models. This prediction can be further analysed by investigating their vorticity prediction. As presented in Figure 22, SBES TSST model predicts stronger vortex shedding at $\theta=75^{\circ}$ resulting in stronger detached flow away from near field of the blade, while other hybrid RANS-LES models (especially DDES and IDDES) still generate attached flow towards near field of the blade.

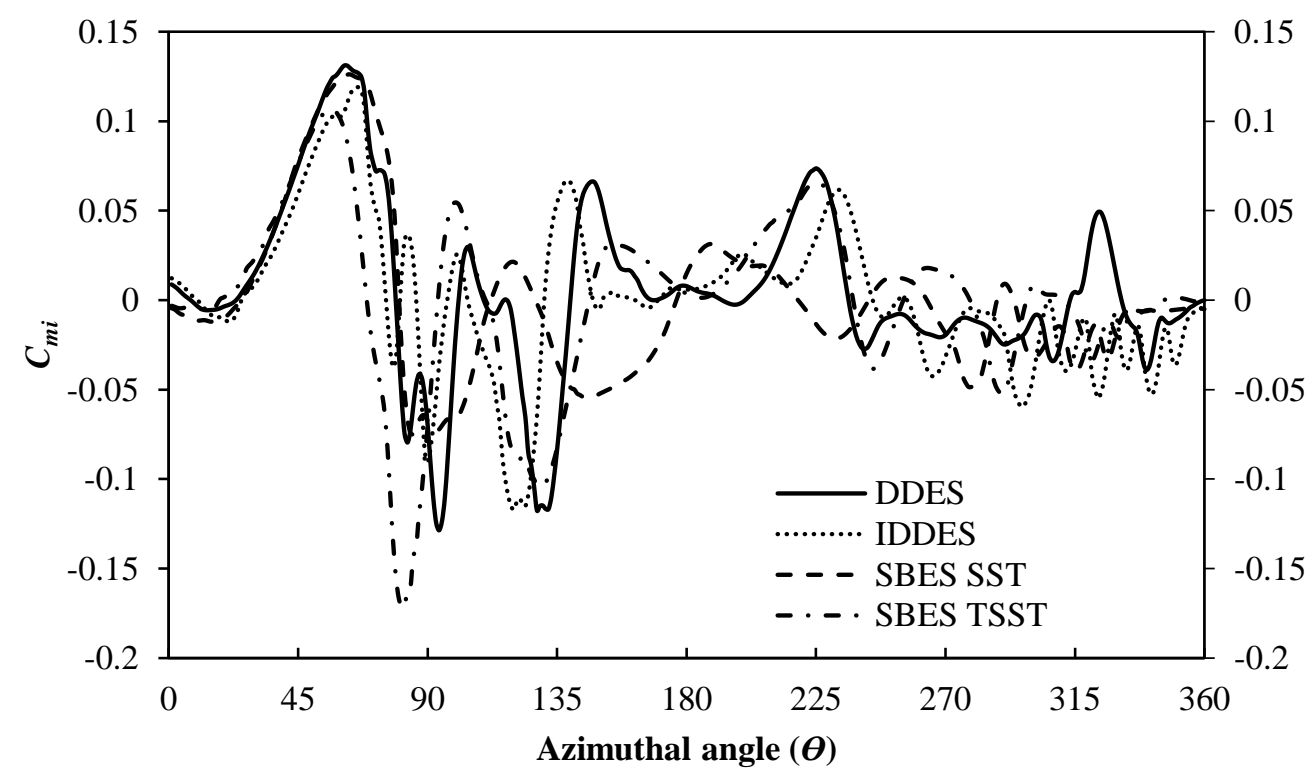

Figure 21. Comparison of instantaneous moment coefficient distribution $\left(C_{m i}\right)$ of four hybrid RANS-LES models (DDES, IDDES, SBES SST and SBES TSST) in TSR $=1.44$.

Furthermore, all hybrid RANS-LES models produce stronger fluctuation in $C_{m i}$ distribution compared to URANS models in low range of TSRs (see Figure 21). This shows that hybrid RANS-LES models can predict stronger vortex shedding (i.e. stronger dynamic stall) than URANS models thereby can generate smaller discrepancy of $C_{\text {pave }}$ prediction compared to experiment data. Vorticity visualisation shows that hybrid RANS-LES models can more reliably generate detached flow away from near field of the blade compared to URANS models (see Figure 22). 


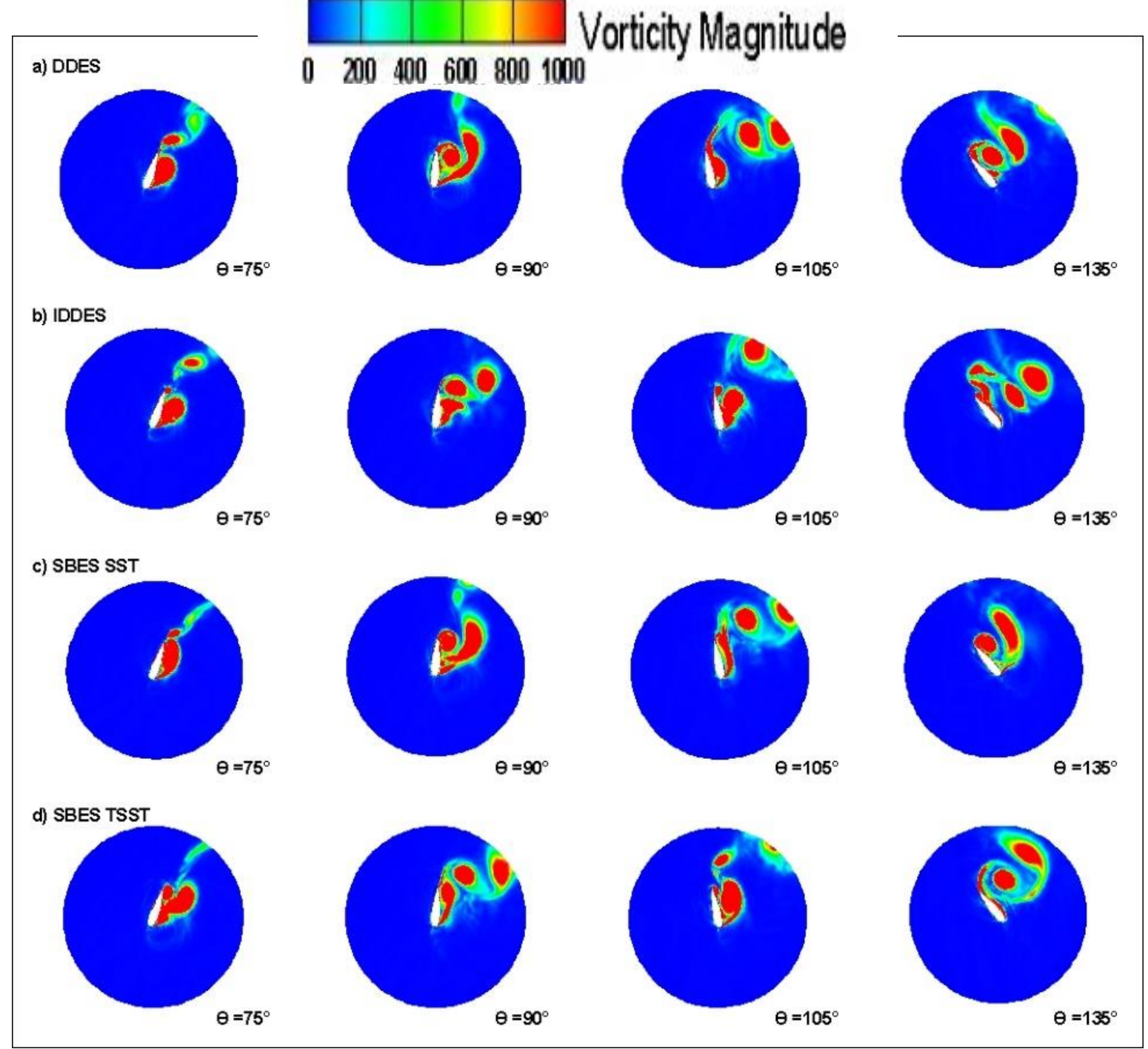

Figure 22. Comparison of contour plots of $z$-vorticity $(\xi)$ indicating the process of flow separation at important azimuthal positions after vortex shedding in $T S R=1.44$ (using hybrid RANS-LES models).

\section{2) Medium range of TSR (Representative TSR $=2.64$ )}

In medium range of TSRs, hybrid RANS-LES models still give different prediction than URANS models but not as severe as in lower TSRs. In regard to prediction of $C_{m i}$ distribution, IDDES and DDES models show stronger fluctuation of $C_{m i}$ distribution than SBES models after the big drop of $C_{m i}$ region (after around $\theta=135^{\circ}$ ) as displayed in Figure 23. Furthermore, hybrid RANS-LES models generate similar peak value of $C_{m i}$ except for IDDES models. SBES TSST model presents latest big fall of $C_{m i}$ but weakest fluctuation in the range of dynamic stall than other hybrid RANS-LES models (i.e. proved by smaller vortex shedding after around $\theta=$ $135^{\circ}$ (see Figure 24)). In addition, it predicts a slower big fall of $C_{m i}$ compared DDES and 
IDDES models. As a result, SBES TSST model generates $C_{\text {pave }}$ lower than DDES and SBES SST models but higher than IDDES model. Nevertheless, it gives the closest prediction to the experimental result.

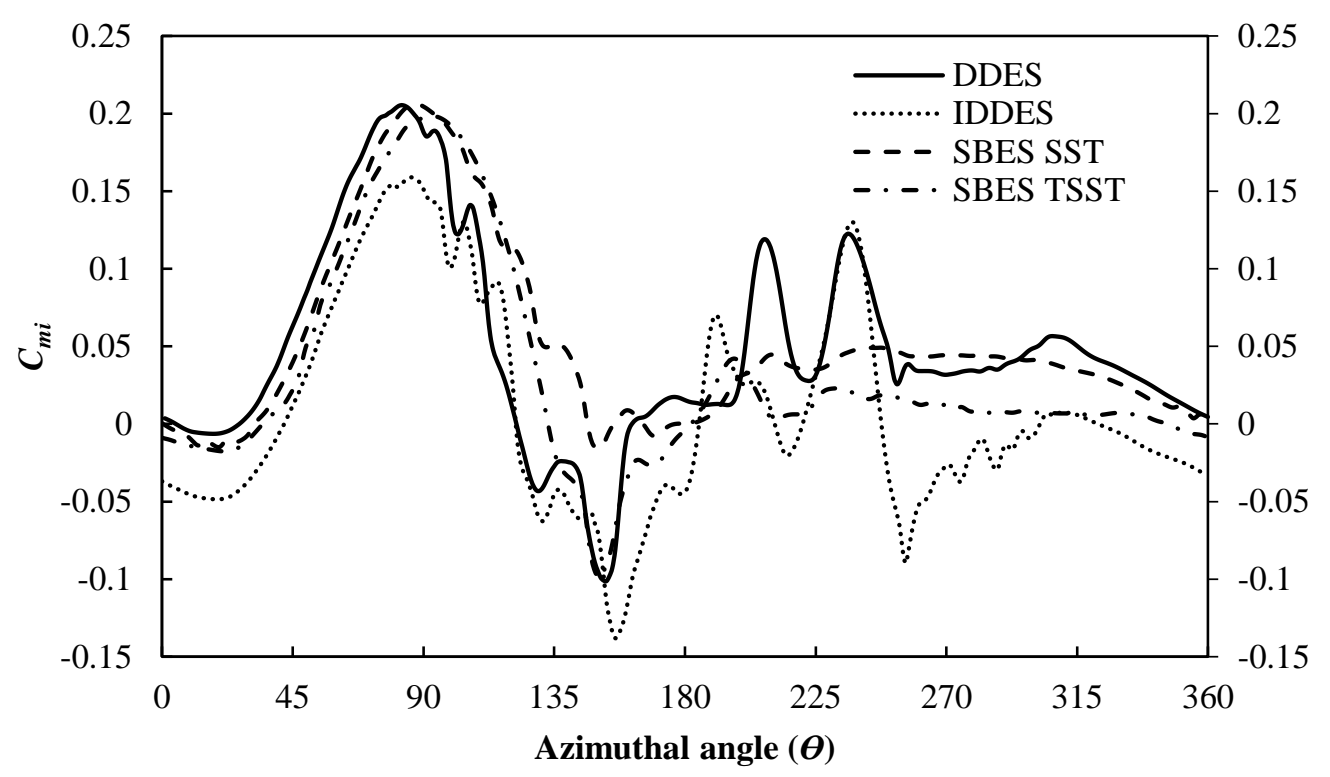

Figure 23. Comparison of instantaneous moment coefficient distribution of four hybrid RANS models (DDES, IDDES, SBES SST and SBES TSST) in TSR $=2.64$. 


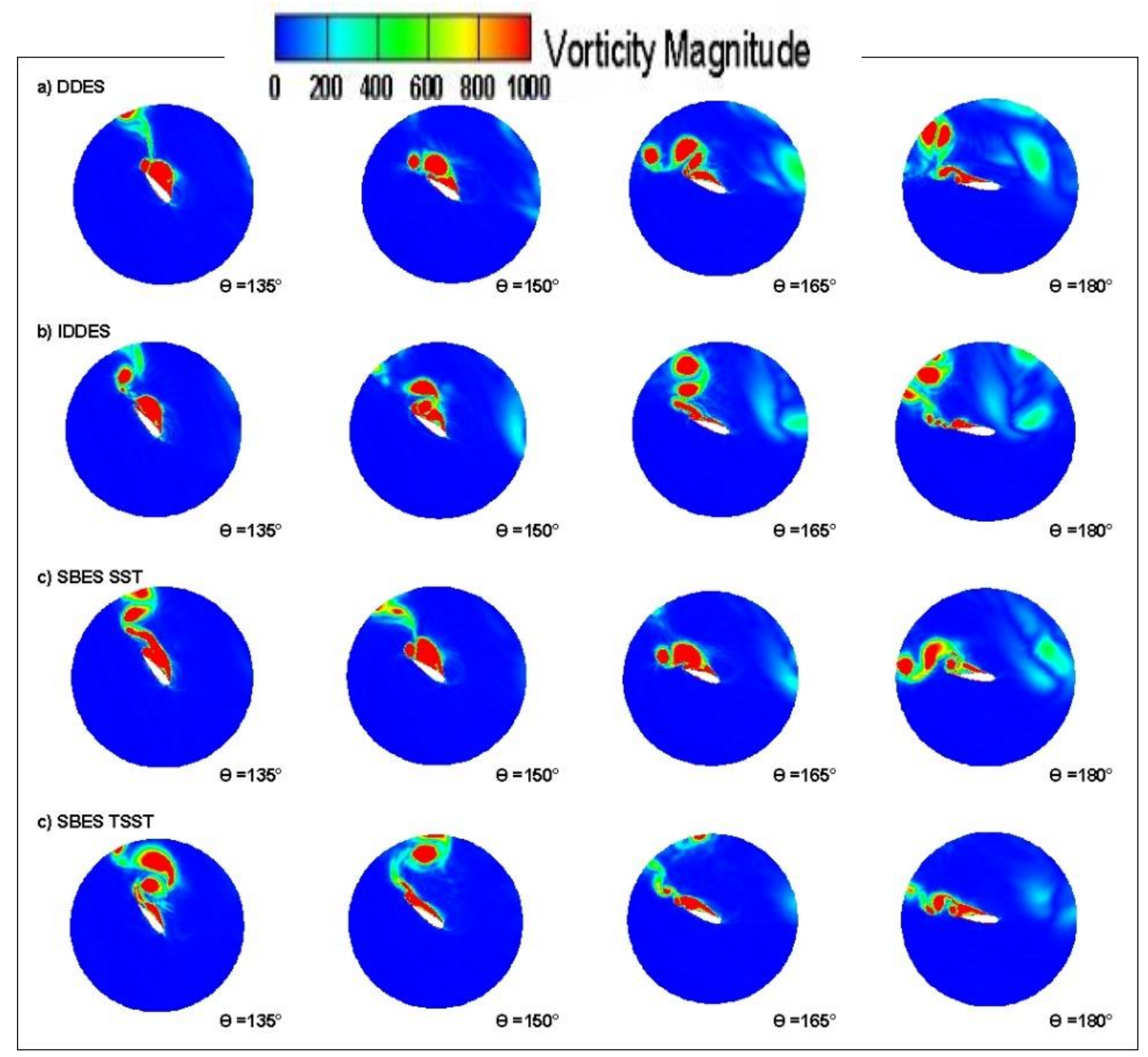

Figure 24. Comparison of contour plots of $z$-vorticity $(\xi)$ indicating the process of flow separation at important azimuthal positions after vortex shedding in TSR $=2.64$ (using hybrid RANS-LES models).

It is noticed that hybrid RANS-LES models show small fluctuation of $C_{m i}$ in the range of big fall of $C_{m i}$ (around $\theta=90^{\circ}-135^{\circ}$ ) compared to URANS models. This indicates that hybrid RANS-LES models can predict stronger vortex shedding in this region than URANS models. This ability is confirmed by prediction of vortex growth of hybrid RANS LES models that demonstrates stronger shedding of vortices than URANS models at $\theta=135^{\circ}$.

3) High range of TSR (Representative TSR $=3.3$ )

As mentioned before, in high TSRs range, URANS models have difficulty to generate accurate prediction even with the use of Transition SST model due to generation of strong vortex shedding in the range of high azimuthal angle was not determined. In this regard, hybrid RANS-LES models show a promising result to address this problem. Compared to URANS 
models, while these models produce relatively smooth distribution of $C_{m i}$ after $\theta=180^{\circ}$, the prediction of $C_{m i}$ distributions by hybrid RANS-LES models demonstrate slight fluctuation in this region (see Figure 25). This shows that hybrid RANS-LES models predict the present of vortex shedding in this region. Vorticity visualisation of hybrid RANS-LES models in this region confirms that there is flow detachment indicating vortex shedding which triggers stronger dynamic stall (see Figure 26).

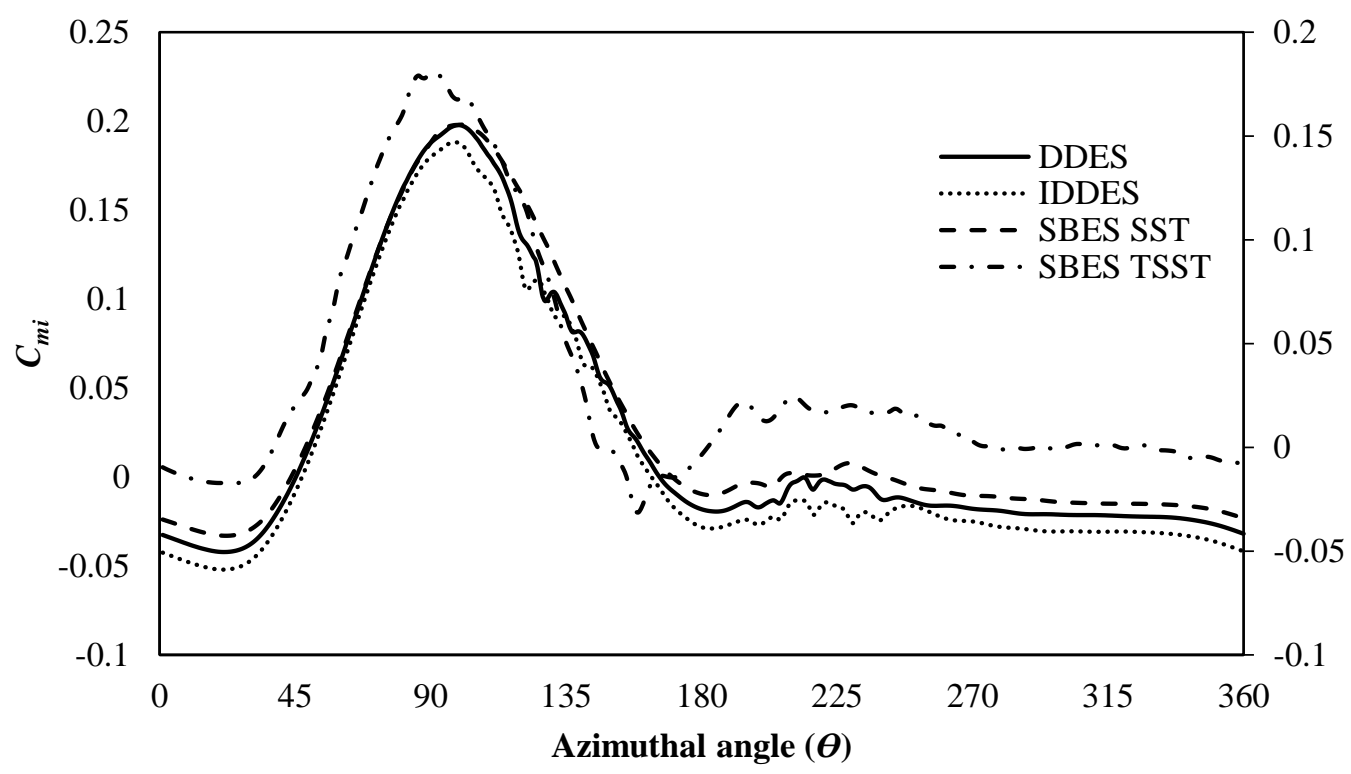

Figure 25. Comparison of instantaneous moment coefficient distribution of four hybrid RANS models (DDES, IDDES, SBES SST and SBES TSST) in TSR $=3.3$. 


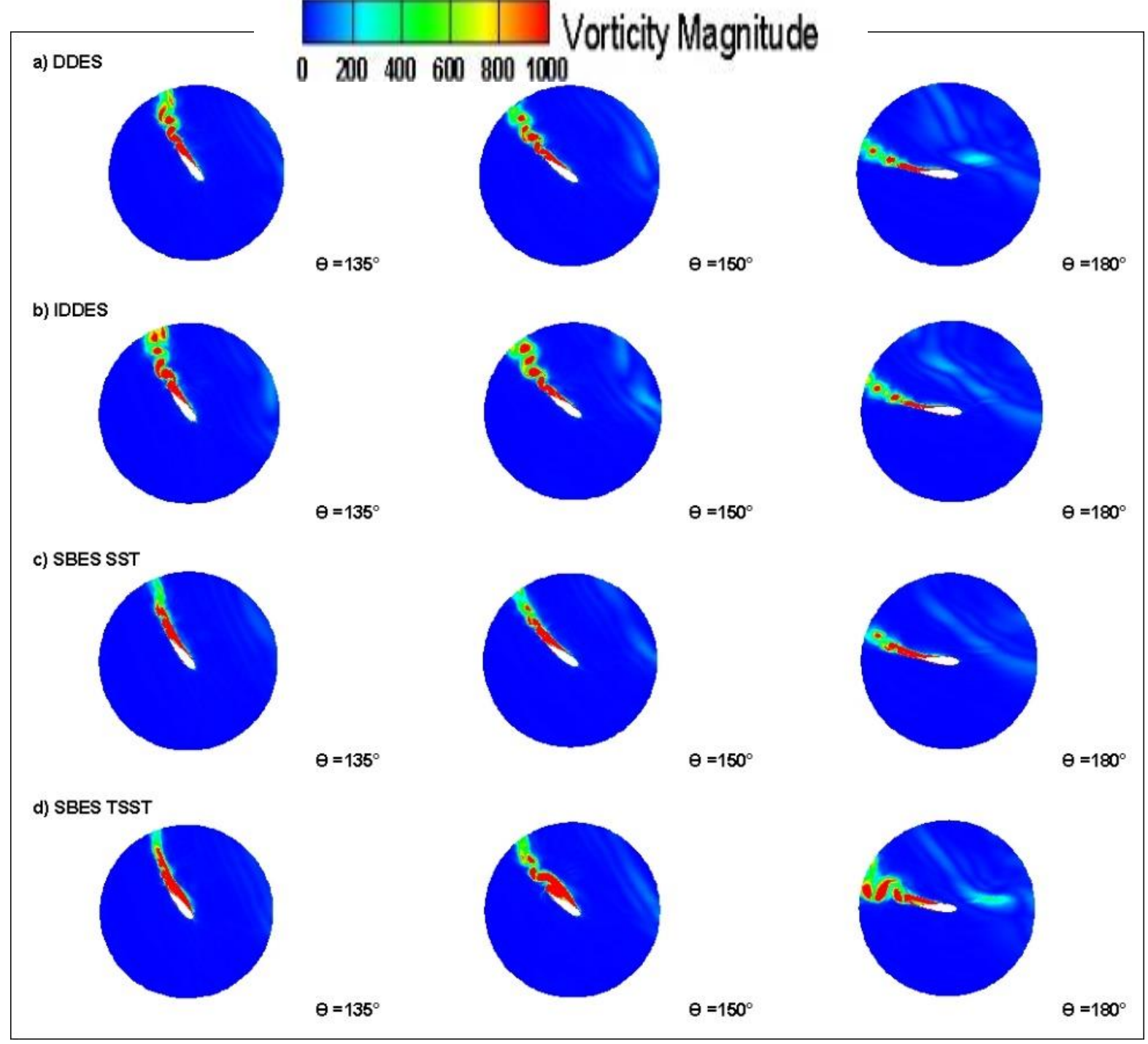

Figure 26. Comparison of contour plots of $z$-vorticity $(\xi)$ indicating the process of flow separation at important azimuthal positions after vortex shedding in TSR $=3.3$ (using hybrid RANS-LES models).

Figure 25 illustrates that SBES TSST model predicts the earliest big drop and the lowest peak value of $C_{m i}$ with quite similar drop rate compared to other hybrid RANS-LES models. Therefore, SBES TSST model can produce the lowest $C_{\text {pave }}$ and it has a closest data match to the experimental data. It is also recognized that SBES models produce weaker fluctuation at the big drop region than DDES and IDDES models indicating that SBES models predict weaker vortex shedding at this region than DDES and IDDES models. This is validated by the prediction of flow behaviour of SBES TSST model that presents weaker leading edge vortex shedding and smaller detached flow at $\theta=135^{\circ}$ (see Figure 26). 


\section{Discussions and Conclusion}

\section{Model Accuracy}

Evaluation of three unsteady RANS turbulence models (i.e. RKE, SST and TSST) and four hybrid RANS-LES models (i.e. DDES, IDDES, SBES with SST $k-\omega$ and SBES with Transition SST) on predicting the performance of three-straight-bladed VAWT is discussed and compared to experiment of Castelli et al. [13]. By referencing to Castelli et al.'s study [13], 2D CFD model is chosen aiming to decrease the computational cost as this study focuses on evaluating the ability of the different turbulence models to predict the flow characteristics around VAWT. Moreover, it was mentioned in the previous studies that 2D computational model of VAWT can give reasonable overall prediction on the performance and flow field around a rotor.

The results show that, in general, two-equation turbulence model produces relatively large discrepancy compared to the experiment data especially in low and medium ranges of TSRs (in which most VAWTs are operated). In regard to the revolution averaged of turbine power coefficient $\left(C_{\text {pave }}\right)$, RKE and SST turbulence models generate around $400 \%$ and $15 \%$ discrepancy in low and medium ranges of TSRs respectively. These discrepancies can be further reduced by using four-equation turbulence models, for example TSST model, which can decrease the discrepancy in low and medium ranges of TSRs to about $100 \%$ and $8 \%$ respectively. Nevertheless, all of RANS turbulence models give similar discrepancy in high range of TSRs (about 17\%). The ability of TSST model to reduce the discrepancy in low and medium ranges of TSRs is contributed by its capability to predict stronger dynamic stall, trailing edge roll up and secondary vortices showed by the presence of vortex shedding in both trailing and leading edges.

Considering relatively low accuracy of RANS turbulence models to predict the performance and flow behaviour of VAWTs and demanding need of computer resources for LES, hybrid RANS-LES turbulence models were examined to analyse their capability to predict the performance and flow behaviour of VAWTs with relatively low to moderate increase in computational resources. This study demonstrates that all four hybrid RANS-LES turbulence models (DDES, IDDES, SBES with SST $k-\omega$ and SBES with Transition SST) have the ability to generate small discrepancy of $C_{\text {pave }}$ prediction in all ranges of TSRs. In low TSRs range, hybrid RANS-LES models can reduce the discrepancy by almost $60 \%$ on average compared to the TSST model. In medium and high TSRs range, the discrepancy reduction varies. While DDES, IDDES and SBES with SST $k-\omega$ models still generate relatively similar discrepancy compared to TSST model (between 5-7\% compared to experimental data), SBES 
with TSST model can produce further discrepancy reduction in medium TSRs range (around 2.2\% compared to experimental data). SBES with TSST model joined by IDDES model also produce small discrepancy (about 2\%) in high TSRs range in comparison with DDES and SBES with SST $k$ - $\omega$ models (approximately 15\%). Hence, SBES with TSST model generates overall the best accuracy in all TSRs ranges compared to URANS and other hybrid RANS-LES turbulence models. Note that, hybrid RANS-LES turbulence models are also better in predicting dynamic stall behaviour compared to RANS turbulence models as indicated by further vortex shedding away from near blade wall. In addition, they can also predict weak trailing edge roll up in high ranges TSRs while RANS turbulence models only show weak shedding on the vortex around trailing edge. Therefore, hybrid RANS-LES turbulence models (in particular IDDES and SBES with TSST) have the capability to generate lower discrepancy in high ranges TSRs.

\section{Rationale for the Choice of Turbulence Models}

Even though SBES with TSST model generates the best accurate results compared to other evaluated models in all TSRs ranges, additional simulation time compared to URANS models needs to be considered (see Table 3) in application, and the time overhead is mostly due to stricter grid requirements (e.g. grid quality). The choice of turbulence model also depends on the VAWT's operation condition that need to be evaluated at both design and off-design points over a wider range. Due to the longer simulation time of hybrid RANS-LES turbulence models and more complex grid generation (for example, SBES TSST model can increase the simulation time by about $25 \%$ compared to TSST turbulence models), URANS models are still the best choice for overall performance evaluation and results produced are quite reasonable in terms of accuracy in specific ranges of tip speed ratios (TSRs). Note that, Transition SST turbulence model is recommended in low to medium TSRs ranges due to relatively good accuracy in these ranges of TSRs as a result of better prediction of the development of dynamic stall compared to RKE and SST models. Compared to RKE and SST models, TSST model gives similar prediction on flow separation in high TSRs range resulting in identical accuracy of $C_{\text {pave }}$ prediction (about $17 \%$ differences compared to the experimental data). However, this model will increase simulation time by around $34 \%$ and $27 \%$ compared to RKE and SST models, respectively. Therefore, beyond the optimum TSR value, the use of RKE or SST turbulence model is acceptable as it produces relatively similar results as TSST model at shorter simulation time. 
Table 3. Comparison of 2D CFD simulation time using different turbulence models.

\begin{tabular}{|c|c|}
\hline Turbulence model & $\begin{array}{c}\text { Total simulation times } \\
\text { (hours) }\end{array}$ \\
\hline RKE & 35.604 \\
\hline SST & 37.26 \\
\hline TSST & 47.628 \\
\hline DDES & 260.304 \\
\hline IDDES & 261.664 \\
\hline SBES SST & 56.304 \\
\hline SBES TSST & 59.296 \\
\hline
\end{tabular}

Nevertheless, if the analysis is taken further to examine the flow behaviour (such as wake development) or noise production and performed in all TSRs ranges, hybrid RANS-LES type of models are recommended, in particular, SBES Transition SST turbulence model as it can produce accurate predictions in all TSRs ranges with reasonable simulation time increment and grid complexity (in average, about $20 \%$ at low TSRs range, $2.2 \%$ at medium TSRs range and $2 \%$ at high TSRs range compared to TSST model, respectively). Note that, the use of DDES or IDDES turbulence model is not recommended as it can increase simulation time by around 4.5 times compared to TSST turbulence model.

\section{Acknowledgement}

The first author would like to acknowledge the Ministry of Research, Technology and Higher Education of Indonesia and Indonesian Endowment Fund for Education (LPDP) of the Ministry of Finance of Indonesia for the financial support through BUDI LPDP scholarship (Reference Letter Number: S-1166/LPDP.3/2017).

\section{References}

[1] L. Wang, L. Zhang and N. Zeng, "A potential flow 2-D vortex panel model: Applications to vertical axis straight blade tidal turbine," Energy Conversion and Management, vol. 47, pp. 454-461, 2007.

[2] H. Beri and Y. Yao, "Double Multiple Stream Tube Model and Numerical Analysis of Vertical Axis W ind Turbine," Energy and Power Engineering, vol. 3, pp. 262-270, 2011.

[3] A. Arab, M. Javadi, M. Anbarsooz and M. Moghiman, "A numerical study on the aerodynamic performance and the self-starting characteristics of a Darrieus wind turbine considering its moment of inertia," Renewable Energy, vol. 107, pp. 298-311, 2017. 
[4] M. Ghasemian, Z. N. Ashrafi and A. Sedaghat, "A review on computational fluid dynamic simulation techniques for Darrieus vertical axis wind turbines," Energy Conversion and Management, vol. 149, pp. 87-100, 2017.

[5] K. Dixon, The Near Wake Structure of a Vertical Axis Wind Turbine, Master Thesis ed., Delft: Delft University of Technology, 2008.

[6] I. Mălăel, H. Dumitrescu and V. Cardoş, "Numerical Simulation of Vertical Axis Wind Turbine at Low Speed Ratios," Global Journal of Researches in Engineering: I Numerical Methods, vol. 14, no. 1, pp. 9-20, 2014.

[7] P. Wernert, W. Geißler, J. Kompenhans and M. Raffel, "Experimental and numerical investigations of dynamic stall on a pitching airfoil," AIAA Journal, vol. 34, no. 5, pp. 982-989, 1996.

[8] A. Rezaeiha, H. Montazeri and B. Blocken, "On the accuracy of turbulence models for CFD simulations of vertical axis wind turbines," Energy, vol. 180, pp. 838-857, 2019.

[9] H. Lam and H. Peng, "Study of wake characteristics of a vertical axis wind turbine by two- and three-dimensional computational fluid dynamics simulations," Renewable Energy, vol. 90, pp. 386-398, 2016.

[10] H. Lei, D. Zhou, Y. Bao, Y. Li and Z. Han, "Three-dimensional Improved Delayed Detached Eddy Simulation of a two-bladed vertical axis wind turbine," Energy Conversion and Management, vol. 133, pp. 235-248, 2017.

[11] H. Peng and H. Lam, "Turbulence effects on the wake characteristics and aerodynamic performance of a straight-bladed vertical axis wind turbine by wind tunnel tests and large eddy simulations," Energy, vol. 109, pp. 557-568, 2016.

[12] "Ansys Fluent User's Guide," ANSYS Inc, [Online]. Available: https://ansyshelp.ansys.com/account/secured?returnurl=/Views/Secured/corp/v194/flu_u g/flu_ug.html. [Accessed 0211 2020].

[13] M. R. Castelli, A. Englaro and E. Benini, "The Darrieus wind turbine: Proposal for a new performance prediction model based on CFD," Energy, vol. 36, pp. 4919 - 4934, 2011.

[14] F. Menter, "Stress-blended eddy simulation (SBES) - A new paradigm in hybrid RANSLES modeling," Notes on Numerical Fluid Mechanics and Multidisciplinary Design, vol. 137, pp. 27-37, 2018.

[15] R. A. Ghazalla, M. H. Mohamed and A. A. Hafiz, "Synergistic analysis of a Darrieus wind turbine using computational fluid dynamics," Energy, vol. 189, paper ID. 116214, 2019.

[16] Y. Wang, S. Shen, G. Li, D. Huang and Z. Zheng, "Investigation on aerodynamic performance of vertical axis wind turbine with different series airfoil shapes," Renewable Energy, vol. 126, pp. 801-818, 2018. 
[17] E. Sobhani, M. Ghaffari and M. J. Maghrebi, "Numerical investigation of dimple effects on darrieus vertical axis wind turbine," Energy, vol. 133, pp. 231-241, 2017.

[18] F. Balduzzi, A. Bianchini, R. Maleci, G. Ferrara and L. Ferrari, "Critical issues in the CFD simulation of Darrieus wind turbines," Renewable Energy, vol. 85, pp. 419-435, 2016.

[19] A. Rezaeihaa, H. Montazeria and B. Blockena, "Towards accurate CFD simulations of vertical axis wind turbines at different tip speed ratios and solidities: Guidelines for azimuthal increment, domain size and convergence," Energy Conversion and Management, vol. 156, pp. 301-316, 2018.

[20] G. Bangga, T. Kusumadewi, G. Hutomo, A. Sabila, T. Syawitri, H. Setiadi, M. Faisal, R. Wiranegara, Y. Hendranata, D. Lastomo, L. Putra and S. Kristiadi, "Improving a twoequation eddy-viscosity turbulence model to predict the aerodynamic performance of thick wind turbine airfoils," in 3rd International Conference on Mathematics: Pure, Applied and Computation, Surabaya, Indonesia, 2017.

[21] T.-H. Shih, W. W. Liou, A. Shabbir, Z. Yang and J. Zhu, "A New - Eddy-Viscosity Model for High Reynolds Number Turbulent Flows - Model Development and Validation," Computers Fluids, vol. 24, no. 3, pp. 227-238, 1995.

[22] T.-H. Shih, J. Zhua and J. L. Lumley, "A new Reynolds stress algebraic equation model," Computer Methods in Applied Mechanics and Engineering, vol. 125, pp. 287-302, 1995.

[23] D. C. Wilcox, Turbulence Modelling for CFD, California, USA: DWC Industries, 2006.

[24] F. Menter, "Two-Equation Eddy-Viscosity Turbulence Models for Engineering Applications," AIAA Journal, vol. 32, no. 8, pp. 1598-1605, 1994.

[25] B. E. Launder and D. B. Spalding, "Lectures in Mathematical Models of Turbulence," Academic Press, London, England, 1972.

[26] M. Kato and B. E. Launder, "The modelling of turbulent flow around stationary and vibrating square cylinders," in Ninth Symposium on "Turbulent Shear Flows, Kyoto, Japan, 1993.

[27] F. Menter, R. Langtry and S. Volker, "Transition Modelling for General Purpose CFD Codes," Flow Tubulence Combust, vol. 77, pp. 277-303, 2006.

[28] R. B. Langtry, F. Menter, S. Likki, Y. Suzen, P. Huang and S. Völker, "A CorrelationBased Transition Model Using Local Variables-Part II: Test Cases and Industrial Applications," Journal of Turbomachinery, vol. 128, no. 3, pp. 423-434, 2006.

[29] R. B. Langtry, J. Gola and F. Menter, "Predicting 2D Airfoil and 3D Wind Turbine Rotor Performance using a Transition Model for General CFD Codes," in 44th AIAA Aerospace Sciences Meeting and Exhibit, Reno, Nevada, USA, 2006.

[30] F. Menter and M. Kuntz, "Adaptation of eddy-viscosity turbulence models to unsteady separated flow behind vehicles," in The Aerodynamics of Heavy Vehicles: Trucks, Buses, 
and Trains. Lecture Notes in Applied and Computational Mechanics, F. B. J. R. R. McCallen, Ed., Berlin, Germany, Springer, 2004, pp. 339-352.

[31] P. R. Spalart, S. Deck, M. L. Shur, K. D. Squires, M. K. Strelets and A. Travin, "A New Version of Detached-eddy Simulation, Resistant to Ambiguous Grid Densities," Theoretical and Computational Fluid Dynamics, vol. 20, pp. 181-195, 2006.

[32] M. Elkhoury, T. Kiwata, K. Nagao, T. Kono and F. ElHajj, "Wind tunnel experiments and Delayed Detached Eddy Simulation of a three-bladed micro vertical axis wind turbine," Renewable Energy, vol. 128, pp. 63-74, 2019.

[33] M. L. Shur, P. R. Spalart, M. K. Strelets and A. K. Travin, "A hybrid RANS-LES approach with delayed-DES and wall-modelled LES capabilities," International Journal of Heat and Fluid Flow, vol. 29, pp. 1638-1649, 2008.

[34] T. Frank and F. Menter, "Validation of URANS SST and SBES in ANSYS CFD for the Turbulent Mixing of Two Parallel Planar Water Jets Impinging on a Stationary Pool Validation of URANS SST and SBES in ANSYS CFD for the Turbulent Mixing of Two Parallel Planar Water Jets Impinging on a Stationary Pool," in ASME 2017 Verification and Validation Symposium, Las Vegas, Nevada, USA, 2017.

[35] S. Ravelli and G. Barigozzi, "Dynamics of Coherent Structures and Random Turbulence in Pressure Side Film Cooling on a First Stage Turbine Vane," Journal of Turbomachinery, vol. 141, no. 1, paper ID. 011003, 2019.

[36] S. Ravelli and G. Barigozzi, "Stress-Blended Eddy Simulation of Coherent Unsteadiness in Pressure Side Film Cooling Applied to a First Stage Turbine Vane," Journal of Heat Transfer, vol. 140, no. 9, paper ID. 092201, 2018.

[37] W. Cai, Y. Li and C. Liu, "Comparative Study of Scale-resolving Simulations for Marine-Propeller Unsteady Flows," International Communications in Heat and Mass Transfer, vol. 100, pp. 1-11, 2019.

[38] M. Mcmullen, A. Jameson and J. J. Alonso, "Acceleration of Convergence to a Periodic Steady State in Turbomachinery Flows," in 39th AIAA aerospace sciences meeting \& exhibit, Nevada, USA, 2001.

[39] S. M. Salim, K. C. Ong and S. C. Cheah, "Comparison of RANS, URANS and LES in the Prediction of Airflow and Pollutant Dispersion," Lecture Notes in Electrical Engineering, vol. 170, pp. 263-274, 2013.

[40] N. Ma, H. Lei, Z. Han, D. Zhou, Y. Bao, K. Zhang, L. Zhou and C. Chen, "Airfoil optimization to improve power performance of a high-solidity vertical axis wind turbine at a moderate tip speed ratio," Energy, vol. 150, pp. 236-252, 2018.

[41] P. R. Childs, "Rotating Cylinders, Annuli, and Spheres," in Rotating Flow, Oxford, Elsevier, 2011, pp. 177-247. 
[42] R. Langtry and F. Menter, "Correlation-based transition modeling for unstructured parallelized computational fluid dynamics codes," AIAA Journal, vol. 47, pp. 2894-2906, 2009.

[43] Z. Warhaft, "Passive Scalars in Turbulent Flows," Annual Review of Fluid Mechanics, vol. 32, pp. 203-240, 2000.

[44] J. M. McDonough, "Introductory Lectures on Turbulence Physics, Mathematics and Modeling," Departments of Mechanical Engineering and Mathematics, University of Kentucky, Kentucky, U.S, 2007.

[45] A. Travin, M. Shur, M. Strelets and P. Spalart, "Detached-Eddy Simulations Past a Circular Cylinder," Flow, Turbulence and Combustion, vol. 63, pp. 293-313, 2000.

[46] M. Ragheb and A. M. Ragheb, "Wind Turbine Theory - The Betz Equation and Optimum Rotor Tip Speed Ratio," in Fundamental and Advanced Topics in Wind Power, Rijeka, Croatia, InTech, 2011, pp. 19-38.

[47] M. Bakırcı and S. Y1lmaz, "Theoretical and computational investigations of the optimal tip-speed ratio of horizontal-axis wind turbines," Engineering Science and Technology, an International Journal, vol. 21, no. 6, pp. 1128-1142, 2018. 